\title{
Traction power substation balance and losses estimation in AC railways using a power transfer device through Monte Carlo analysis
}

\author{
Vítor A. Morais ${ }^{1}$ (D) António P. Martins ${ }^{1}$
}

Received: 25 June 2021 / Revised: 8 October 2021/Accepted: 10 October 2021/Published online: 17 January 2022

(C) The Author(s) 2022

\begin{abstract}
The high dynamic power requirements present in modern railway transportation systems raise research challenges for an optimal operation of railway electrification. This paper presents a Monte Carlo analysis on the application of a power transfer device installed in the neutral zone and exchanging active power between two sections. The main analyzed parameters are the active power balance in the two neighbor traction power substations and the system power losses. A simulation framework is presented to comprise the desired analysis and a universe of randomly distributed scenarios are tested to evaluate the effectiveness of the power transfer device system. The results show that the density of trains and the relative branch length of a traction power substation should be considered in the evaluation phase of the best place to install a power transfer device, towards the reduction of the operational power losses, while maintaining the two substations balanced in terms of active power.
\end{abstract}

Keywords Electric traction systems - Monte Carlo analysis - Power transfer device - Power quality - Railway power systems $\cdot$ Smart railways

Vítor A. Morais

vitor.fa.morais@gmail.com

António P. Martins

ajm@fe.up.pt

1 Department of Electrical and Computer Engineering, Faculty of Engineering, University of Porto, Porto 4200-465, Portugal

\section{Introduction}

Electrified railways have constantly raised interest since they are considered one of the most energy-efficient modes of transportation. It is known from the report of [1] that the railway sector is efficient when compared to other means of transportation: the railway sector has a $9 \%$ market share in the transportation of passengers and goods in the European Union and this is achieved with a final energy consumption of $2 \%$, in comparison with other sectors. The considerable power requirements of the railway transportation system lead the railway operators to pursue actions to increase the energy efficiency, reduce the energy consumption bill and increase the infrastructure capacity, [2, 3].

This work is framed within the IN2STEMPO ${ }^{1}$ project objectives-Innovative Solutions in Future Stations, Energy Metering and Power Supply-specifically in the smart power supply activities. Inserted in the Shift2Rail European program [4], the smart power supply activities seek to contribute to the development of a railway power grid in a fully interconnected and communicating system, [5]. In the smart power supply target of IN2STEMPO, one goal is to study Flexible AC Transmission Systems (FACTS) to exploit the full potential of the $25 \mathrm{kV}, 50 \mathrm{~Hz}$ rail power supply system. Specifically, one of the FACTS under study is the Railway Interline Power Flow Converter (RIPFC) [6]. The RIPFC comprehends a power transfer device (PTD) in the neutral zone (NZ), providing doubleside feeding to the catenary branch and respective trains, and reactive power on both sides to stabilize the catenary voltage.

The objective of this paper is to statistically evaluate the effects of the inclusion of a PTD in the neutral zone of an

\footnotetext{
${ }^{1}$ https://cordis.europa.eu/project/id/777515.
} 
electrified railway line. Mainly, the active power balancing in the two traction power substations (TPS) and the system power losses are under consideration in this study.

The first contribution of this paper is a simulation framework to statistically evaluate the proposed PTD compensation strategy (where the framework is capable to be extended to additional statistical analysis). Then, the second contribution is the evaluation of two scenarios, with different PTD characteristics, when they are considered design constraints (namely, the catenary branch length and the sparsity/density of trains in the TPS).

This paper is divided into seven sections. Sect. 2 addresses a scientific literature review on the topologies for power transfer devices and on the control strategies. Section 3 presents the architecture for the simulation model necessary to implement the required analysis. Then, Sect. 4 covers reduced scenario simulations to illustrate the advantages of this strategy, in terms of active power balancing and system power losses. Section 5 covers the universe of parameters randomly analyzed with the Monte Carlo analysis methodology. Section 6 presents the results and the discussion on the. Finally, the conclusions are presented in Sect. 7.

\section{Literature review}

Electrified railways, DC or AC, must deal with several power quality issues, either in the railway subsystem itself and in the transmission/distribution system operator (TSO/DSO) supply [7-10]. In the past, some passive solutions helped to alleviate these issues but their performance was dependent on different operation parameters and has poor dynamics. Then, the use of power electronics based converters for power quality increasing in railway systems has entered into the domain and is continuously increasing [11-13]. Their purpose is quite large and addresses different power quality issues in railway systems: transformer losses, catenary voltage stability, substation active power balancing, peak shaving, three-phase current balancing, reactive power compensation [13-15]. It should be mentioned that the static frequency converter (SFC) system is a kind of "one fits all" regarding all the referred power quality issues but this system is a stand-alone solution, interesting for new lines, [12, 16-18]. Since the large majority of railway systems is based on some type of transformer topology, the SFC system will not be further considered in this paper.

Modern railway line voltage stabilization is made using a static VAR compensator (SVC), located near the neutral zone $[19,20]$, or using mobile reactive power compensation strategies $[3,21,22]$. The railway power conditioner system (RPC) is employed in conjunction with V/V transformers and offers very good performance regarding 3-phase power balancing and power factor correction
[10, 23, 24]. Similarly, the co-phase system [25-28] , has similar characteristics in terms of an optimized 3-phase interface. Maintaining the objective of guaranteeing a high level of power quality at the 3-phase supply, additional solutions are employed, e.g. the STATCOM [29-31].

In recent years, a strong effort is being made to introduce renewables and storage in railway systems. This tendency intends the satisfaction of several main objectives: i) alleviate the substations load and then avoid peak loads (peak shaving) and, at the same time, reduce the system losses [32-34]; ii) turn the railway system an even more environmental friendly [35]; and iii) provide the means for regenerative energy storage $[36,37]$.

There are many factors such as weather conditions, driving style, schedule, and stop time in the stations which alter the actual characteristics of train circulation concerning pre-defined ideal conditions. All of these factors have some randomness degree [38].

The position in the sector, speed and acceleration of the train define both the consumed power and the voltage drop in the line and respective losses and the power factor in the substation. Therefore, it is not possible to carry out a fully deterministic analysis of the various operating parameters of the railway subsystem $[39,40]$.

Thus, several aspects of the power flow analysis regarding both the railway substations and the catenary are better characterized from a probabilistic analysis [41-43]. Analyses of feeder current profile, [44, 45], harmonic propagation in DC [46] or AC railways [47], and the voltage profile in the catenary $[39,48]$, have been performed using probabilistic methods. The analysis of data from both real exploration and dedicated simulators allows defining the probability density functions associated with factors that contain a probabilistic character such as the number of trains in a section, the position, the consumed power, or the power in the substation [41, 49].

The PTD is an additional element in the railway system and the analyses of its effects, both on the balance of active power in substations and on the redistribution of power losses in the system, are the main objectives of this article. As, the railway system becomes more complex, data clustering and probabilistic analysis are used, in particular, the Monte Carlo method. The following section addresses the subsystem models, with particular focus on the developed simulation framework.

\section{Subsystem models}

Let us consider a generic $1 \times 25 \mathrm{kV}, 50 \mathrm{~Hz}$ double-track railway line as illustrated in Fig. 1 including a PTD in the ND. In this configuration, and commonly, each traction 
Transmission system operator: three phase grid, $50 \mathrm{~Hz}$

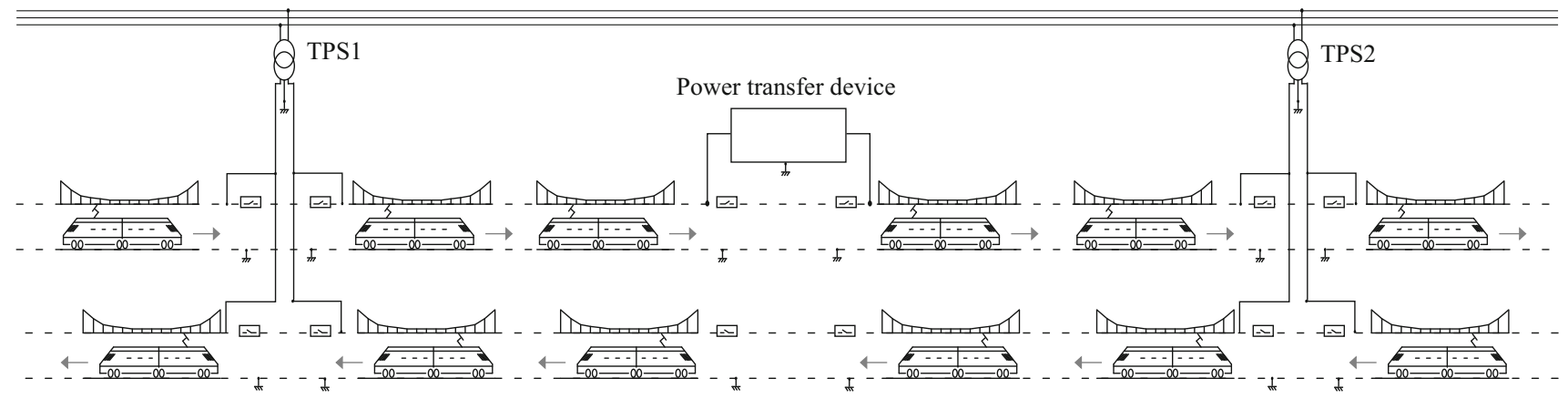

Fig. 1 Simplified representation of the inclusion of a power transfer device on a neutral zone

substation is electrically isolated from an adjacent one where this electric separation is made in the ND.

This paper considers the inclusion of a power converter (a PTD) in the ND, having a back-to-back configuration, as illustrated in Fig. 2, being capable of independently control the reactive power injected in each side of the two sections $\left(Q_{1}\right.$ and $\left.Q_{2}\right)$ and transfer active power between the two sides $\left(P_{\text {ptd }}\right)$. To limit the size of the problem, the work in this paper is only focused on the management of active power where the power factor of the PTD is unitary.

The specific converter structure can be based on different approaches but the most common is a multilevel one, built with cascaded H-bridges, neutral-point clamped, or modular multilevel converters. The transformers are designed according to the power level to be transferred between sections, the reactive power injected in the ND, and the voltage and current levels in the converters. Also, a parameter of high relevance in the system design either in the transformer and in the converters is the efficiency level. This parameter ensures that the power losses value is kept at a minimum and thus does not compromise the overall losses when using the PTD, particularly in the active power transfer mode.

\subsection{Architecture of the simulation framework}

The simulation model of a railway power system is a particular power flow analysis, where the electric model is dynamic. Specifically, the electric model depends on the number of trains and the position of each one (absolute/ relative position). Then, the power flow analysis of a railway power system is a snapshot simulation, where each simulated scenario has a specific matrix for the branches and the buses of the power system.

This work requires the development of a simulation framework, available as open-source in the repository ${ }^{2}$. The simulation framework purpose is to dynamically

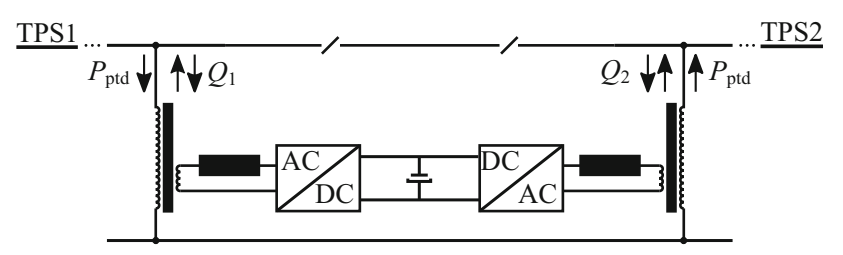

Fig. 2 Simplified architecture of the electronic converter to perform the power transfer device function

generate the branches and bus matrices of the power system based on the definition of the physical conditions. Then, this framework has two functional highlights: for each scenario, the physical characteristics can be established (either fixed or randomly defined), with the automatic power system modeling, and the scenario can be automatically evaluated (using a power flow solver).

The implementation approach depends on the usage of an object-oriented language, where each simulation scenario follows a class of objects capable to implement the functional highlights.

The desired functionality was modeled in a MATLAB simulation framework where the power flow analysis is made using the MatPower tool. The simulation framework has the following functional requirements:

1. Must be capable of simulating all trains of two or four branches of each traction substation (respectively for single and double track lines).

2. Must be implemented in an object-oriented approach, enabling the analysis of an array of simulation scenarios targeting multi-core parallelization. Each simulation scenario is one object, having intrinsic properties and methods.

3. Each simulation scenario (or object) must consider:

- All traction substations - TPS $[1,2, \ldots, T]$ - in adjacent pairs;

- All branches of each TPS - br[1,2] or br[1, 2, 3, 4];

$\overline{{ }^{2} \text { Repository: }}$ github.com/vitormorais/proj_railway_ptd. 
- All trains in each branch of each TPS $T[1,2, \ldots, N]$;

- All power transfer devices existing in the whole line in the analysis - PTD $[1,2, \ldots, P]$.

4. The essential methods of the class must be:

- Dynamic setup of all TPS branches;

- Dynamic addition of $N$ trains to a branch (placed in specific positions, with specific apparent power consumption);

- Dynamic setup of power transfer devices;

- Power flow analysis and automatic output generation;

- Automatic graphical generation of the electric diagram.

Let us consider a simple scenario having two traction substations, each one feeding a single-track $1 \times 25 \mathrm{kV}$ railway line, where both TPS are interconnected through a power transfer device, and the respective two branches (20 km and $15 \mathrm{~km}$, respectively) have three trains each in different positions and with different apparent power. This scenario can be simulated in the proposed simulation framework. Then, the automatic graphical generation output is illustrated in Fig. 3.
The output visible in Fig. 3 can be automatically generated by the simulation framework.

Since there are no trains in the left branch of the TPS1, the branch impedance (br[2]: 2-3) corresponds to the impedance of a $20 \mathrm{~km}$ catenary line. The traction power transformer of TPS1 is represented by branch br[1]: 1-2. The automatic electric model generator ensures that the sum of the impedances of the right branch is equal to the total impedance of a $20 \mathrm{~km}$ catenary line $(\mathrm{br}[7]+\mathrm{br}[8]+$ br[9] $+\operatorname{br}[3]=\operatorname{br}[2])$.

Regarding the PTD, the adopted model comprises two series branches with a middle bus representing the constant losses. The first branch (br[13]: 4-100) represents the series impedance of the PTD step-down transformer; the subsequent branch (br[14]: 100-101) is associated to the inverter conduction losses. The constant power losses in bus 100 represent the transformer constant power losses (no-load losses) together with the inverter constant power losses.

The simulation framework follows the UML diagram of Fig. 4. Each electric_model object has several properties (like the characteristics of the TPS, the information of train positions and respective power consumptions, the characteristics of the PTD, and the relevant parameters for the

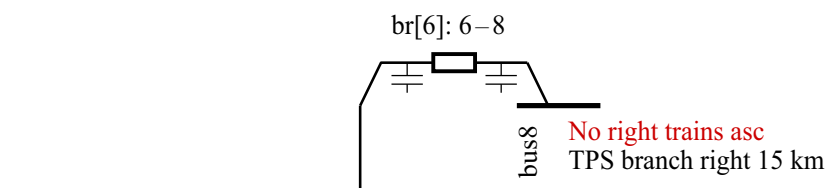

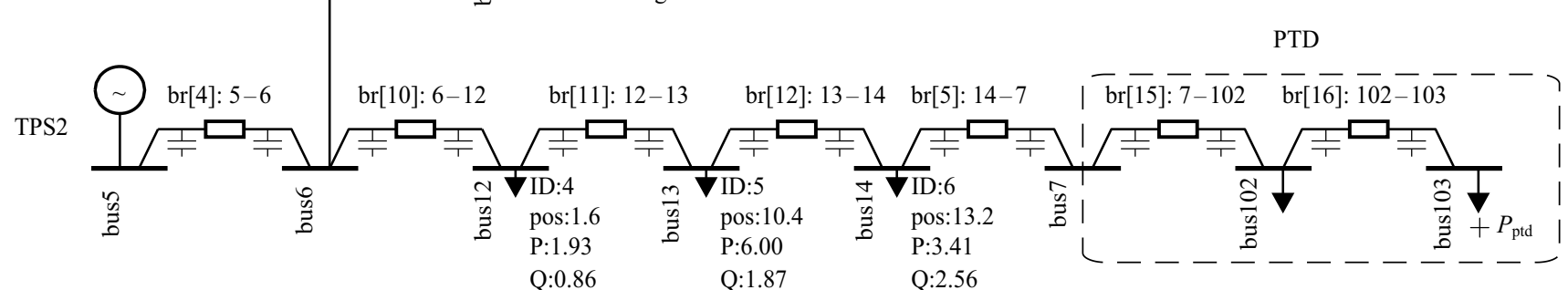

Left trains asc

TPS branch length $15 \mathrm{~km}$

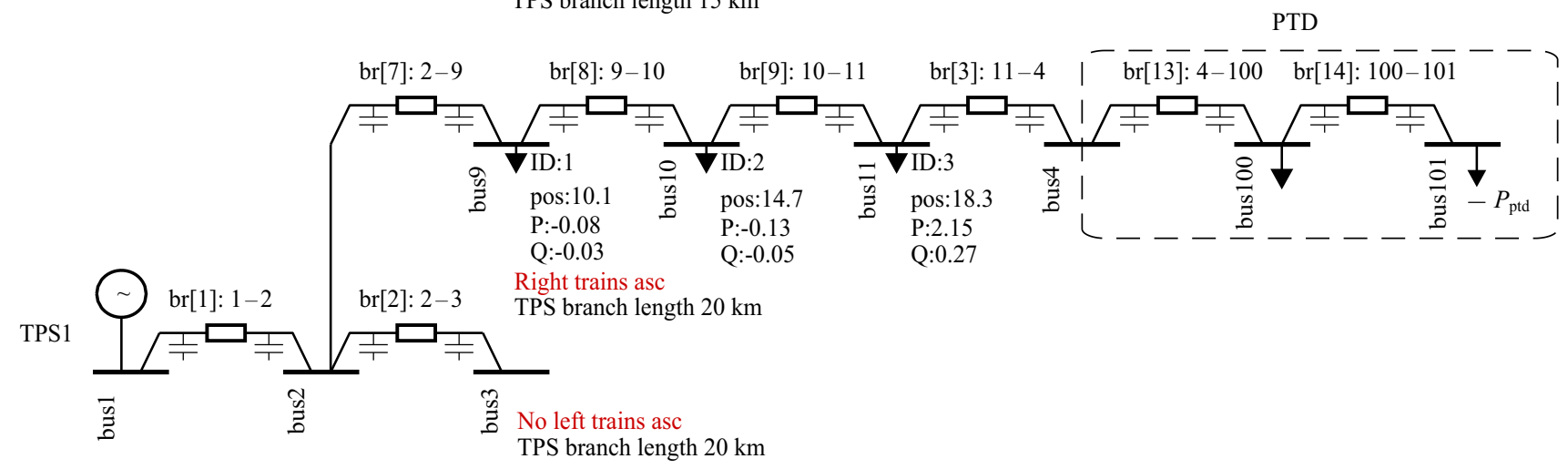

Fig. 3 Resultant implemented simulation model of the simple scenario ( $\mathrm{P}$ stands for active power with units in MW, and Q reactive power with units in MVAr, while pos means position with units in $\mathrm{km}$, and asc ascending direction.) 
power flow solver). Also, each electric_model object has several methods, which are the functions of the class, with examples like: i) electric_model::generate_random_trains() to start a new scenario with random train positions and respective power consumptions; ii) electric_model::run_powerflow () to perform the power flow analysis of the scenario; or iii) electric_model:: attach_power_transfer_device() to include a PTD to the scenario for optimization of the TPS power consumption. Additional methods were also developed to load and save the simulation state, or to plot the resulting electric diagram of the scenario.

A new scenario is evaluated with the sequential launch of the methods of electric_model, as illustrated in Fig. 5 . The initial step is to configure the electric parameters and the two TPS under study. Then, the trains are generated (number of trains, active power, and power factor of each one) for both TPS and the power flow is calculated. Finally, the PTD is attached to the simulation framework with a specific optimization strategy, the power flow is launched again and the final results are extracted. This procedure is then evaluated as a scenario of two TPS without and with the PTD in the ND.

In the example of the application of the flowchart of Fig. 5, the main objective is to balance the active power in each TPS, with the compensation power in the power transfer device being defined by the method electric_model::tps_power_optimization(), where half of the power is transferred in the NZ. In this flowchart, the first part (in green) is to prepare the object for the power flow analysis: first is defined the electric parameters (TPS traction transformer impedance, catenary impedance, among others),

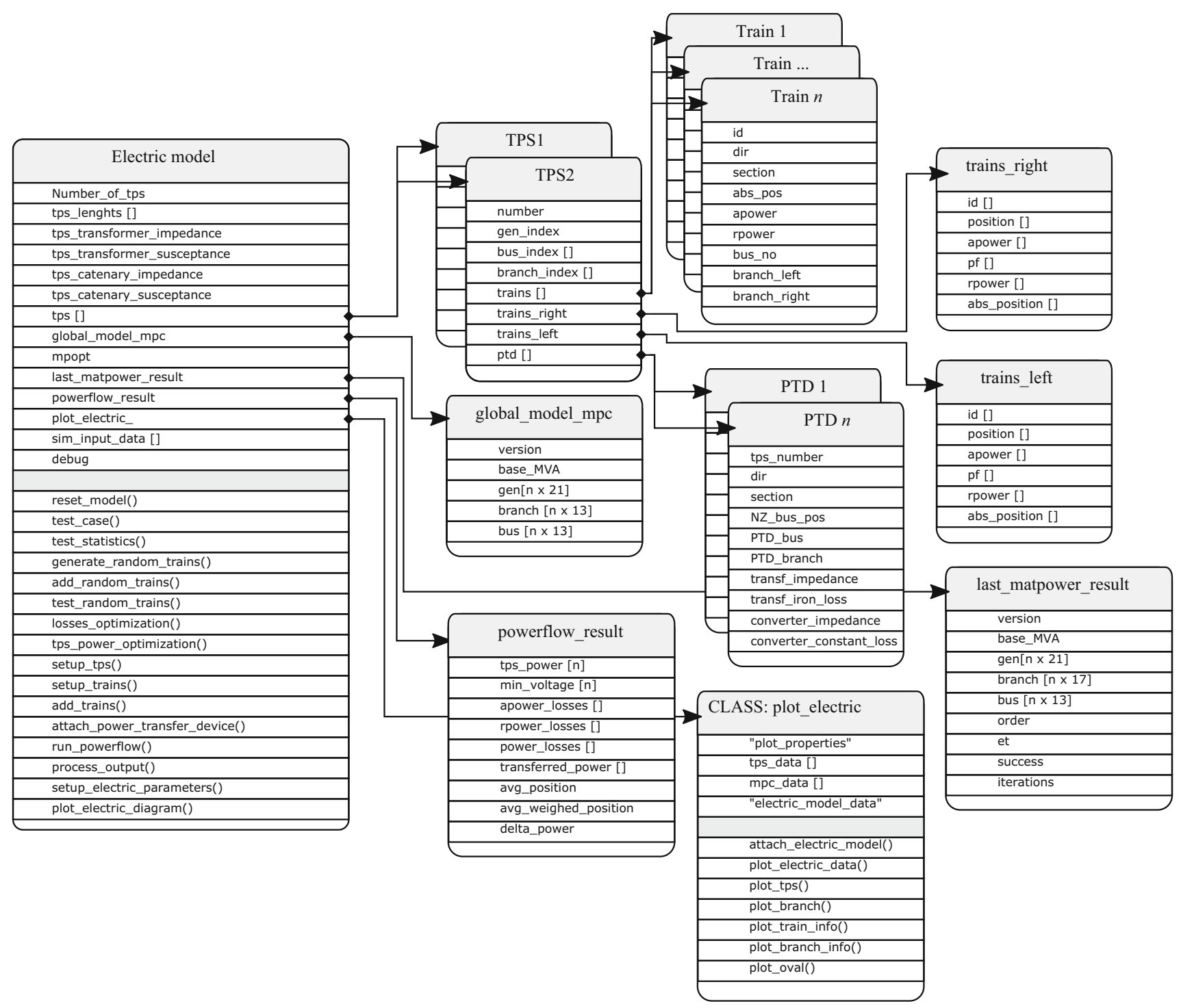

Fig. 4 UML diagram of the resultant implemented simulation model 


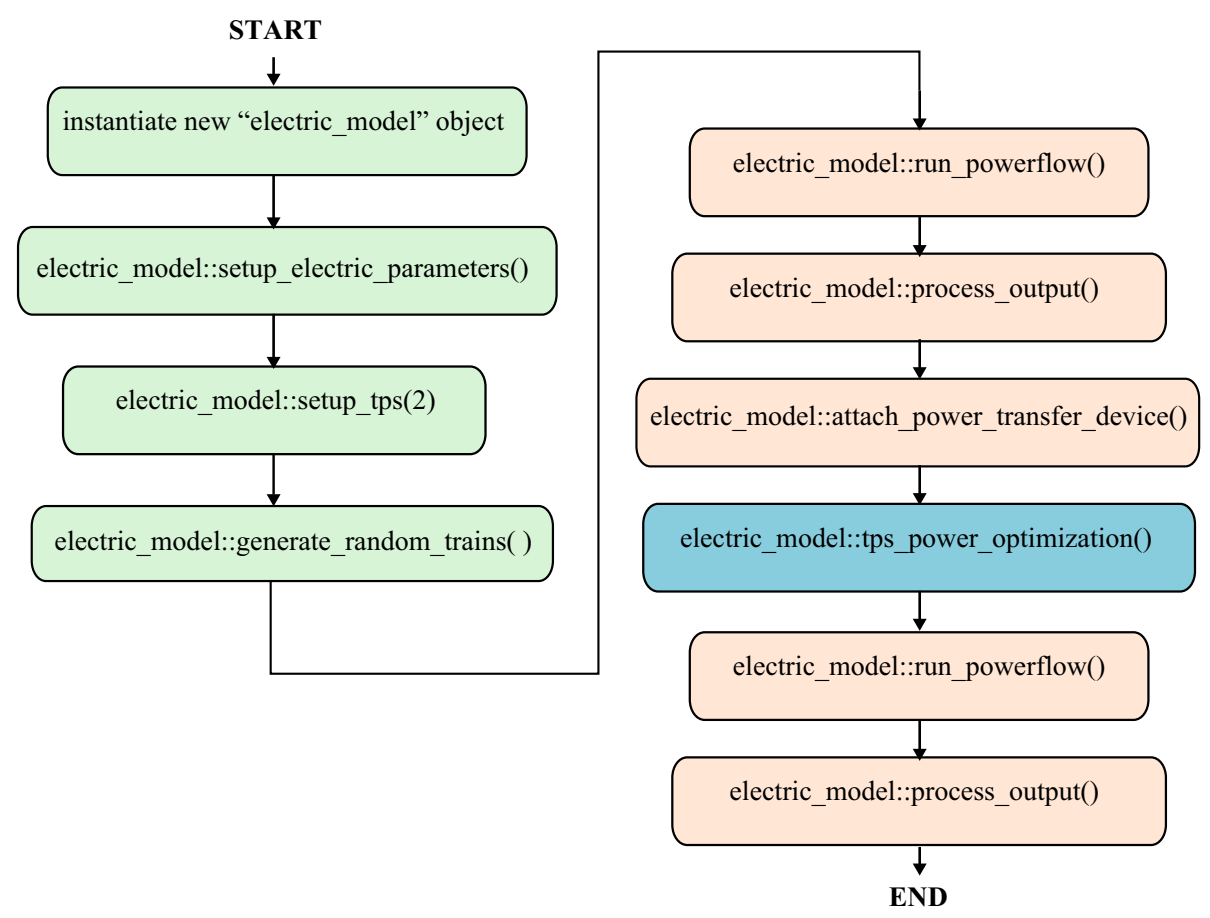

Fig. 5 Example of usage of an electric_model object to evaluate a new scenario

and then is created a new physical simulation scenario (where the number of trains can be random or fixed, as well as the respective apparent power and position in the branches, among other properties). The second part (in coral and blue) targets the specific simulation presented in this paper: the evaluation of the installation of the PTD in the NZ, in terms of power losses, where is obtained the knowledge of the system state before and after the installation.

To evaluate a universe of simulation scenarios, a second handler class was developed, which follows the UML of Fig. 6.

The purpose of this handler class is to hold the array of electric_model_objects and to extract the results on the performed analysis. The main method of this class is the eval_compensation(type,max_iter), where all the new simulation scenarios are created, the random and fixed parameters of each scenario are generated and the power flow analysis is performed, following the flowchart of Fig. 5.

This section was detailed with the implementation steps of the simulation framework capable to evaluate the inclusion of the PTD for a universe of simulation scenarios. The Appendix section is also presented two details of the graphical user interface, in Figs. 26 and 27, where the scenario random generation and the electric parameters menus are presented, respectively.

\section{Reduced scenario simulation}

Before entering a large-scale simulation analysis, it is useful to analyze and validate some simple scenarios, in which the quantitative and qualitative analysis should validate a priori known conclusions.

This section evaluates two sets of simulation scenarios, starting with scenarios comprising two TPS and one train, and then scenarios having two TPS and two trains (each one supplied by a different TPS), using the simulation framework presented in the previous section.

Fig. 7 illustrates a simple simulation of one train and two TPS connected by a PTD.

Let us consider the following reduced scenario analysis, consisting of $N$ scenario simulations, as in (1), where the power consumption and position of this train are randomly distributed following specific probability distribution functions (PDF).

$$
\left\{\begin{array}{cc}
P_{\text {train }}[1,2, \ldots, N] \in[08] & \text { in } \mathrm{MW} \\
D_{\text {train }}[1,2, \ldots, N] \in[01] \quad \text { in } \% \text { of } d_{\text {cat,total }}^{\text {tss } 1}
\end{array} .\right.
$$

For all $N$ scenarios, the train power factor is fixed $\left(p f=0.98\right.$ ind .), the branch lengths are fixed $\left(d_{\text {cat,total }}^{\text {tp1 }}=30\right.$ $\left.\mathrm{km} ; d_{\mathrm{cat}, \text { total }}^{\mathrm{tp} 2}=30 \mathrm{~km}\right)$, the parameters of the TPS $\left(Z_{\mathrm{tps}}\right)$ and the catenary are fixed $\left(R_{\mathrm{cat}}^{*}=0.15 \Omega / \mathrm{km}, \quad L_{\mathrm{cat}}^{*}=\right.$ $1.43 \mathrm{mH} / \mathrm{km} \quad$ and $\quad C_{\text {cat }}^{*}=12.4 \mathrm{nF} / \mathrm{km}, \quad$ where $Z_{\text {cat }}=Z_{\text {cat }}^{*} d_{\text {cat,total }}^{\text {tps }}$ ), and the parameters of the PTD are fixed 


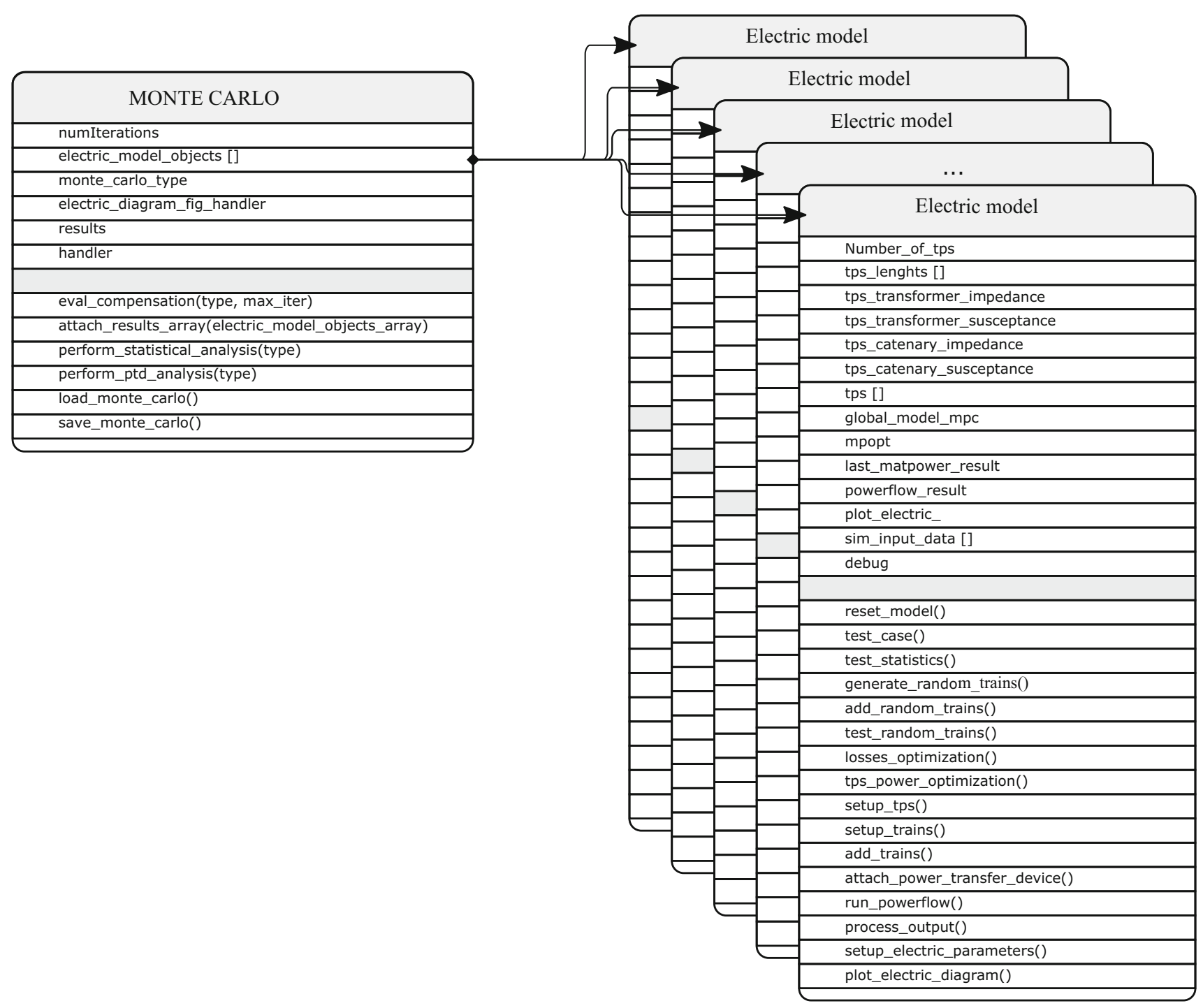

Fig. 6 UML diagram of the resultant implemented simulation model

$\left(R_{T, \mathrm{ptd}}=0.47 \Omega\right.$ and $X_{T, \mathrm{ptd}}=0.94 \Omega$ for the PTD transformer, $R_{I, \mathrm{ptd}}=0.47 \Omega$ and $L_{I, \mathrm{ptd}}=0 \mathrm{mH}$ for the PTD inverter, and the no-load power losses are zero).

Considering that the PTD operates to perfectly balance the traction power between the two TPS, then the formulation of the system power losses can be illustrated in Fig. 8.

Assuming $\left|V_{\mathrm{s} 1}\right|=\left|V_{\mathrm{s} 2}\right|, \quad Z_{\mathrm{tps} 1}=Z_{\mathrm{tps} 2}$ and near-equal power factors, with a perfect power balance obtained with the PTD, the train current is divided in half, and with the same catenary length of the two branches, this results in the invariant power losses of Fig. 9 when it concerns to the $\alpha$ value.

This constant power losses, $P_{z_{l}+z_{r}}$, are the sum of the catenary, TPS and PTD losses, where half of the train current is associated to the $Z_{\mathrm{tps}}+\alpha Z_{\text {cat }}$ impedances, and the other half of current passes through the $Z_{\text {tps }}+Z_{\text {cat }}+$ $2 Z_{\text {ptd }}+(1-\alpha) Z_{\text {cat }}$ impedances.

Then, if the train is only supplied by the TPS 1 with a current $I_{\mathrm{s} 1}$, the power losses can be estimated:

$P_{\text {loss }}^{\text {left }}=\left|I_{\mathrm{s} 1}\right|^{2}\left(R_{\mathrm{tps}}+\alpha R_{\mathrm{cat}}\right)$.

On the other side, if the PTD forces the division of the currents in the two TPS, where $I_{\mathrm{s} 1}=I_{\mathrm{s} 2}=I / 2$, and considering unitary power factors in TPS 1 and TPS2, then:

$P_{\mathrm{loss}, \mathrm{ptd}}^{\mathrm{left}}=\left(\frac{|I|}{2}\right)^{2}\left(R_{\mathrm{tps}}+\alpha R_{\mathrm{cat}}\right)$, 
$P_{\text {loss }, \mathrm{ptd}}^{\mathrm{right}}=\left(\frac{|I|}{2}\right)^{2}\left(R_{\mathrm{tps}}+(1-\alpha) R_{\mathrm{cat}}+R_{\mathrm{cat}}+2 R_{\mathrm{ptd}}\right)$.

The intersection point, where $P_{\text {loss }}^{\text {left }}=P_{\text {loss,ptd }}^{\text {left }}+P_{\text {loss,ptd }}^{\text {right }}$, can be obtained as following:

$\alpha_{0}=\frac{-R_{\mathrm{tps}}+R_{\mathrm{cat}}+R_{\mathrm{ptd}}}{2 R_{\mathrm{cat}}}$.

Then, for the considered assumptions, this results in $\alpha_{0} \approx 0.6$. This value means that the adoption of a PTD with the objective of power balancing leads to a minimization of the system power losses if the train is located between $\alpha_{0}$ and the NZ. The closer the train is to the NZ, the higher the advantages in terms of the system losses, as expected.

Fig. 10 presents the evaluation of the difference of the system power losses for the $N$ scenarios of (1). Each point of the graph corresponds to a simulated scenario (without and with the PTD), and the lines represent the equipotential values where the power losses difference ("after-before" the PTD) is the same. Thus, negative values mean that the PTD action reduced the power losses in the system.

In the simulation results of Fig. 10, it is visible that the actual $\alpha_{0}$ value is close to the theoretic value of $\alpha_{0} \approx 0.6$, from expression in (5). This result is obtained with the balancing in the two TPS visible in Fig. 11.

The previous reduced scenario analysis is useful to evaluate the advantages expected from the inclusion of the PTD. First, it should be highlighted that the major compensation strategy is in the balancing of the two TPS. The example presented can reduce an unbalance from $8 \mathrm{MW}$ to a maximum of $0.45 \mathrm{MW}$ (with the majority of the unbalance values closer to $0 \mathrm{MW}$, thus a perfectly balanced system in terms of power consumption).

(a)

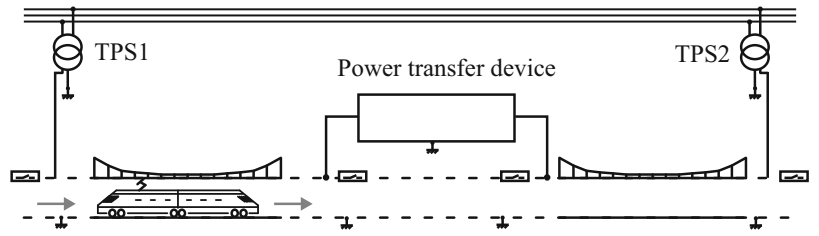

(b)

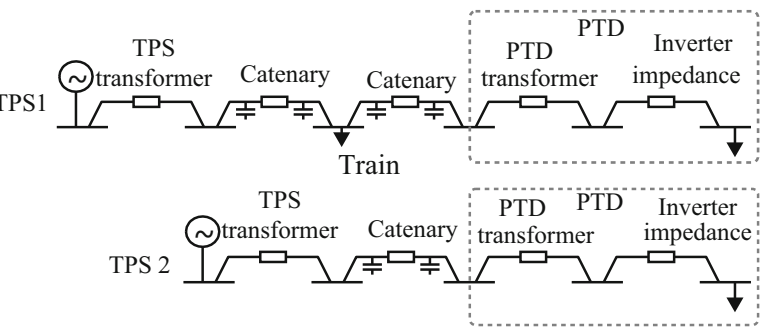

Fig. 7 Illustration of the reduced scenario simulation: a physical representation; b electric diagram

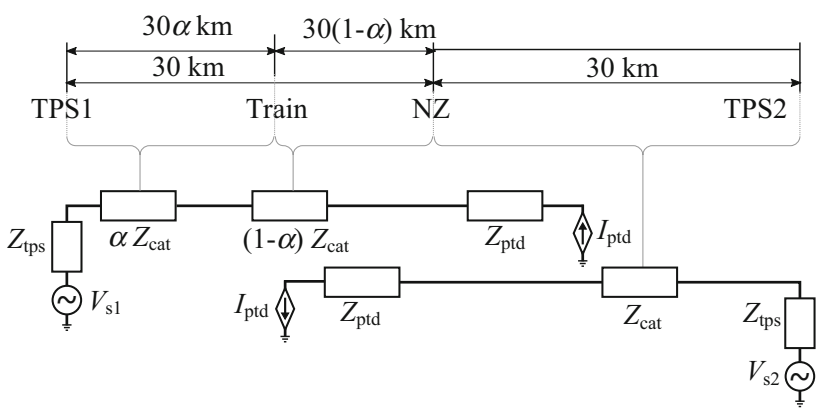

Fig. 8 Formulation of the electric diagram for the one-train reduced scenario analysis

Fig. 12 presents the results for a new testbed of scenarios, where are considered two trains in each TPS branch in addition to the parameters of the previous reduced scenario simulation. Both trains of all $N=10000$ scenarios follow the expression in (1).

The results after the inclusion of the PTD show final power unbalance between the two TPS $( \pm 0.45 \mathrm{MW}$, corresponding to $5.5 \%$ of the maximum transferred power), which are caused by the compensation strategy of the PTD. Essentially, the simulation framework performs an initial power flow analysis for both TPS without the influence of the PTD. Based on the difference of power in the two TPS, this difference is used as a setpoint for the power transfer device and the simulation framework is subject to a final power flow analysis. Only with an iterative process is possible to reduce the final unbalance, which, in terms of implementation, means the need for a feedback loop if a perfect balance is required.

A preliminary analysis of the system power losses before and after the inclusion of the PTD might not justify the adoption of the power converter. Only in a hypothetical scenario where the majority of trains are located near the NZ (where $\alpha>\alpha_{0}$ ) is justified the adoption of PTD. For obvious reasons, the train position follows a uniform PDF

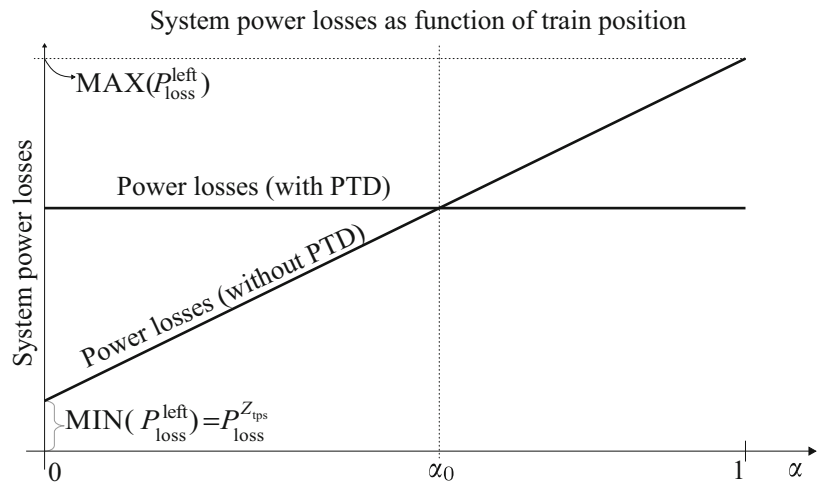

Fig. 9 Power losses evaluation with and without the inclusion of a PTD, for the one-train reduced scenario analysis, as function of the normalized train position $(\alpha)$ 
across the entire railway line. Then, as illustrated by Fig. 10, the majority of the scenarios increase in the system power losses, when comparing the power losses before and after the inclusion of the PTD.

When considering the PTD constant power losses, the results of Fig. 10 are affected by an offset. For example, if these power losses are higher than 0.15 MW, all of the simulation scenarios increase the system power losses.

This section leads to addressing the two metrics of analysis when is considered the inclusion of the PTD: the system power losses and the minimization of unbalance in the TPS power. For the following sections, any discussion on results must consider an analysis on both metrics. As an example, even if every scenario of analysis increases the system power losses, the advantages of adopting a PTD lie in balancing both TPS.

\section{Monte Carlo analysis}

This section presents the details of the simulation scenarios, specifically on the input parameters. Considering the diagram of Fig. 1, a simplified analysis of the adoption of a PTD (to improve the railway infrastructure) can consider two catenary branches of two subsequent TPS.

The universe of simulation scenarios must cover several parameters variation, such as:

- branch number of trains, $\in \mathbb{N}$;

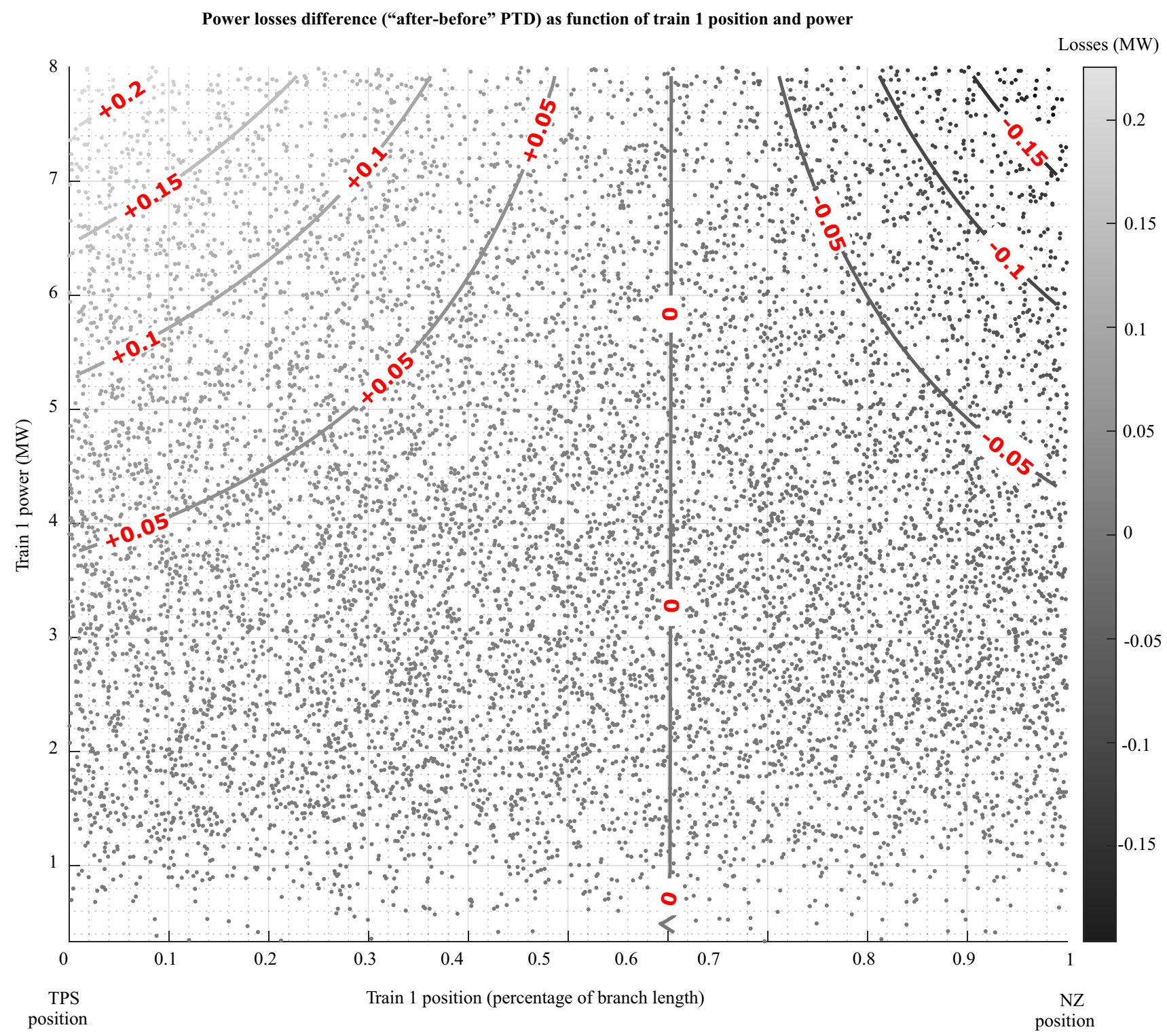

Fig. 10 System power losses difference before and after the inclusion of the PTD, as function of the train position and power consumption with 10000 scenarios simulated 
(a)

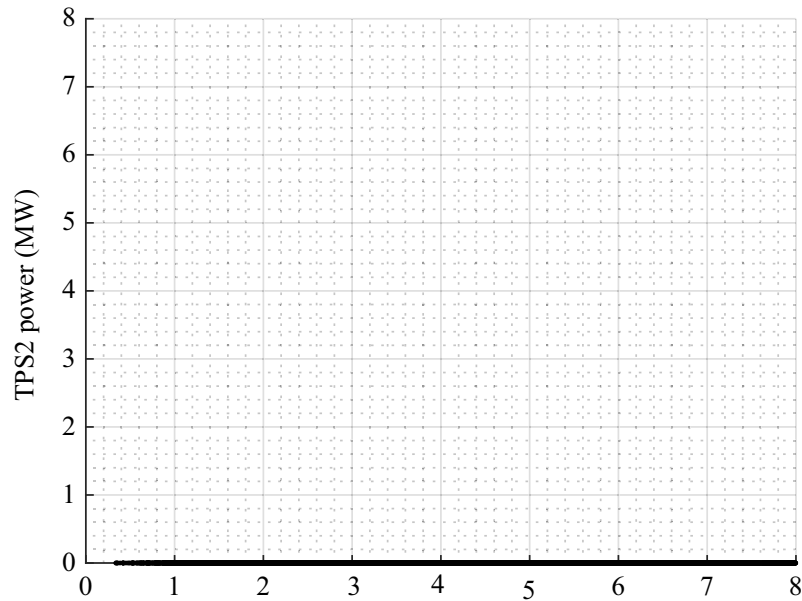

(c)

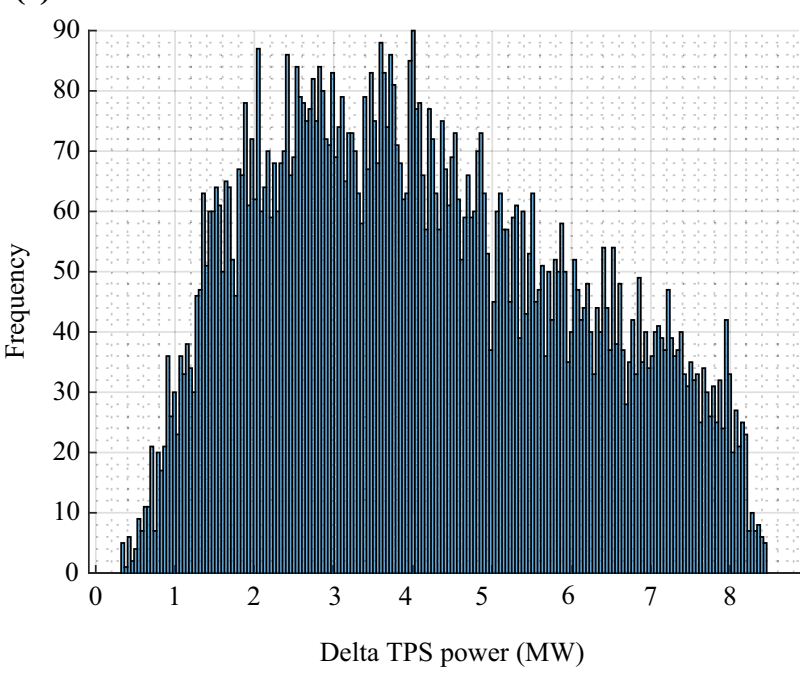

(b)

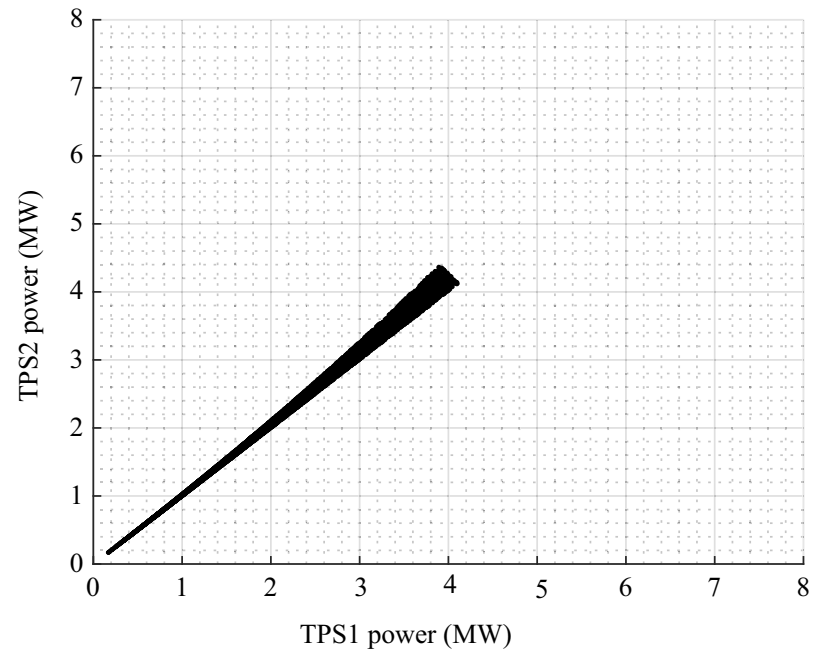

(d)

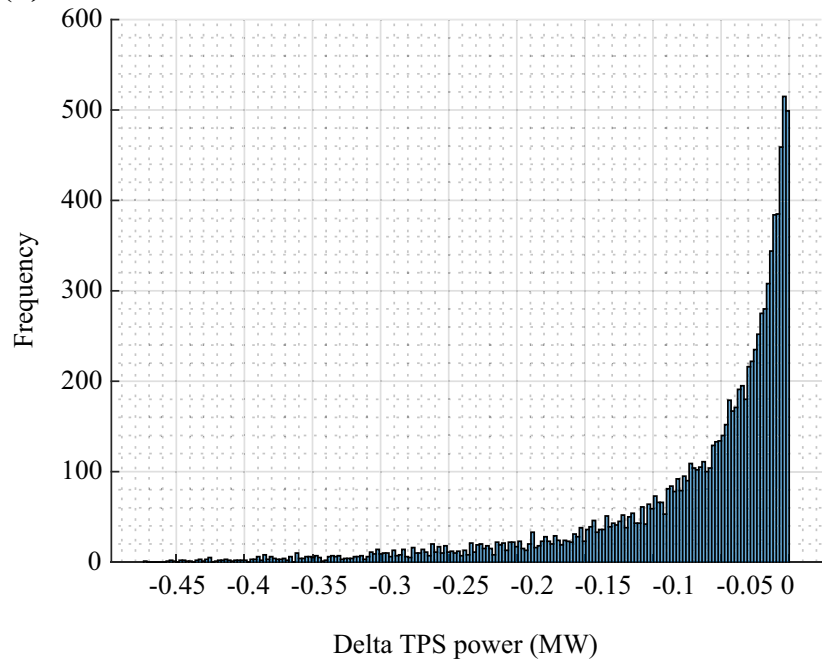

Fig. 11 Simulation results for the $N=10000$ scenarios of Eq. (1): a active power in TPS1 and TPS2 before the inclusion of PTD; b Active power in TPS1 and TPS2 after the inclusion of PTD; $\mathbf{c}$ histogram analysis of the difference of power between TPS1 and TPS2; $\mathbf{d}$ histogram analysis after the inclusion of PTD

- branch length, $\in \mathbb{R}$;

- $\quad$ TPS power profile, $\in \mathbb{R}$;

- train power consumption, $\in \mathbb{R}$;

- train power factor, $\in \mathbb{R}$;

- train position, $\in \mathbb{R}$;

The surface of this universe of simulation scenarios is, at least, $S \in \mathbb{N R}^{5}$.

To reduce the universe of simulation scenarios, some assumptions must be made based on the Monte Carlo statistical analysis. Being the Monte Carlo term associated with the process of modeling and simulating a system that is affected by randomness [50], this analysis requires that each of the parameters follows an appropriate probability distribution functions (PDF).
With the generation of several random scenarios (where the parameters follow PDF functions), and the collection of relevant statistics, thus it can be assessed the performance of a decision policy or the value of an asset [50], or in this work, the decision of the installation of a PTD. The usage of a specific railway line and a dataset of measurements enable the fitting of the PDF for each of the parameters, as shown in the following statistical analysis.

\subsection{Statistical analysis}

In the following, it is considered the relevant characteristics of a $250 \mathrm{~km}$ railway line with seven substations. The first aspect analyzed in this work is on the catenary branch 
(a)

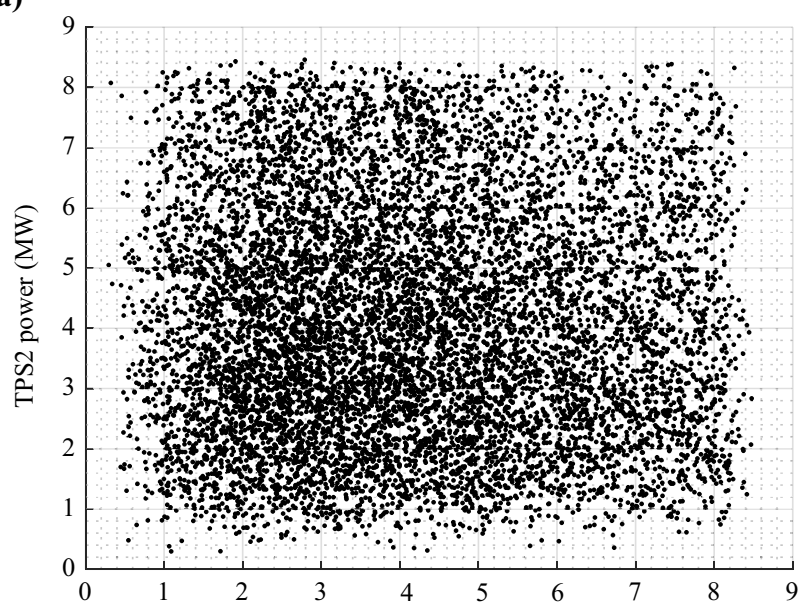

(c)

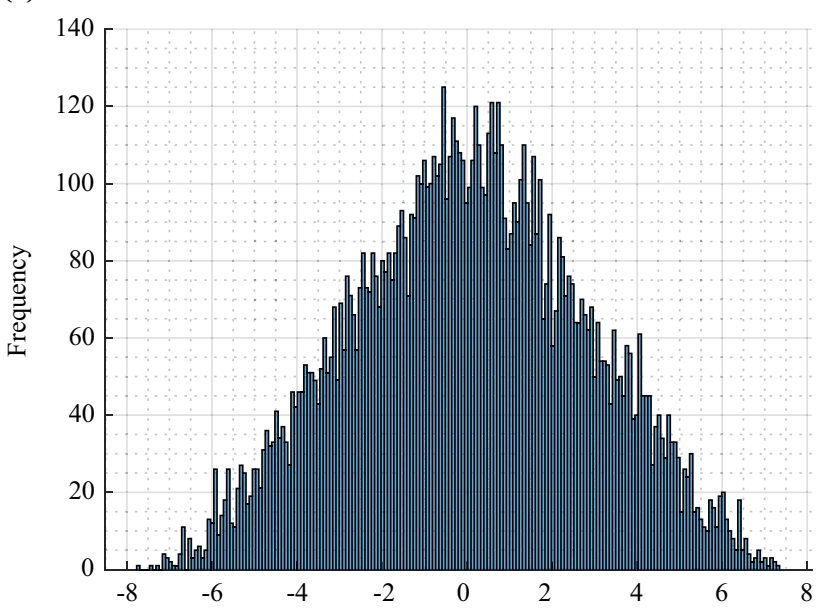

Delta TPS power (MW) (b)
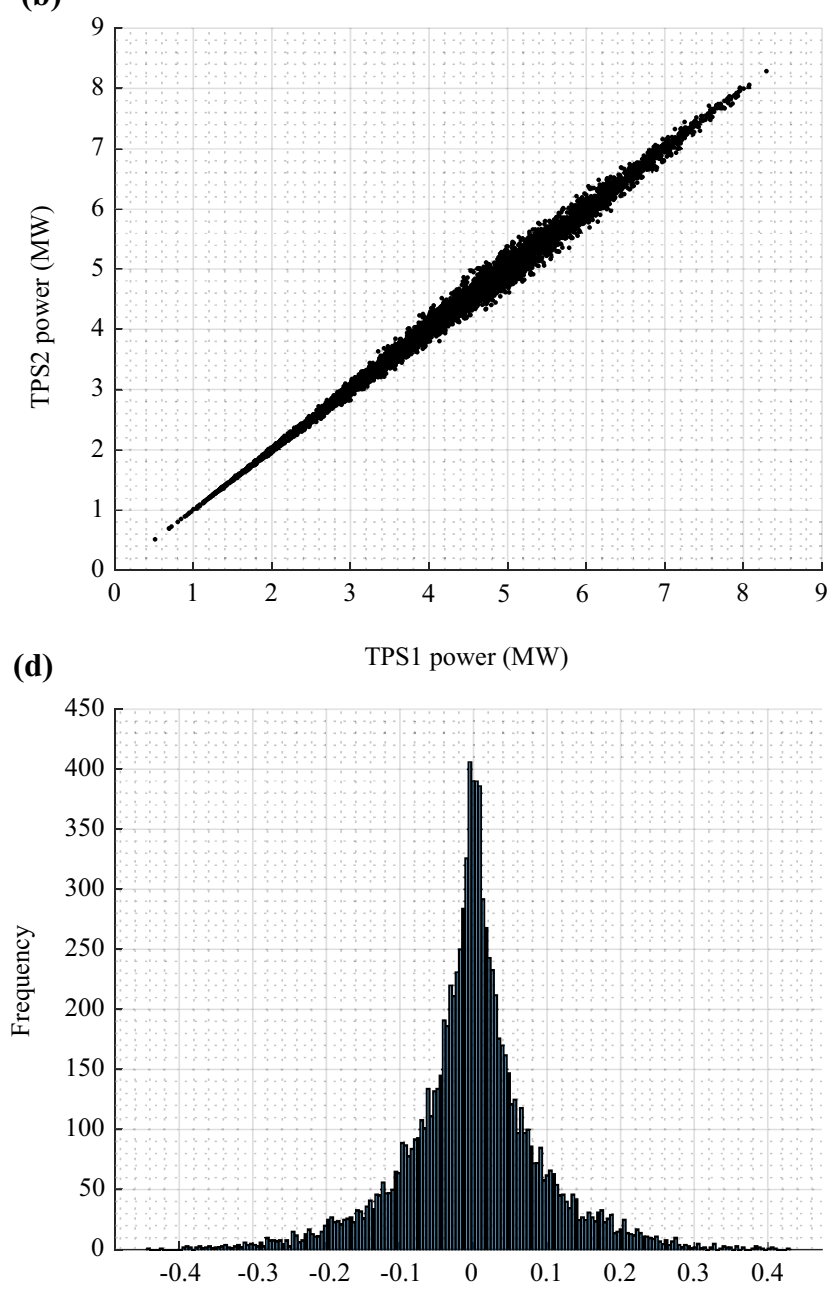

Delta TPS power (MW)

Fig. 12 Simulation results for $N$ scenarios of Eq. (1) for two trains: a active power in TPS1 and TPS2 before the inclusion of PTD; b Active power in TPS1 and TPS2 after the inclusion of PTD; $\mathbf{c}$ histogram analysis of the difference of power between TPS1 and TPS2; $\mathbf{d}$ histogram analysis after the inclusion of PTD

length, where the distance of each branch is visible in Fig. 13.

The branch distance of Fig. 13 does not follow a specific PDF: it could be highlighted that the catenary branch distance is uniformly distributed between $6 \mathrm{~km}$ and $30 \mathrm{~km}$.

Despite the uniform distribution of the length of the catenary branch, it would be interesting the analysis of clustered lengths. It could be argued that long lines can have much more branches and, then, a normal PDF function would be appropriate. Then, in this work, two clusters of line lengths can be defined, where $\mu$ is the average and $\sigma$ is the standard deviation, following:

- Short catenary branch lengths, following a normal PDF with $\mathcal{N}(\mu=15, \sigma=2.5)$;
- Long catenary branch lengths, following a normal PDF with $\mathcal{N}(\mu=21, \sigma=2.5)$.

Statistical analysis for the respective clusters is presented in the histogram graphs of Fig. 14.

Furthermore, this work considers two types of TPS, each of them having a randomly defined number of trains, and a randomly defined power consumption profile.

The first assumption is to define clusters of types of TPS. The rationale behind this assumption is the following: the number of trains throughout the day will define the power consumption requirements of this TPS. For example, a TPS covering a dense metropolitan area will be more likely to have more trains and higher power consumption than a TPS covering a sparse rural area. 
(a)

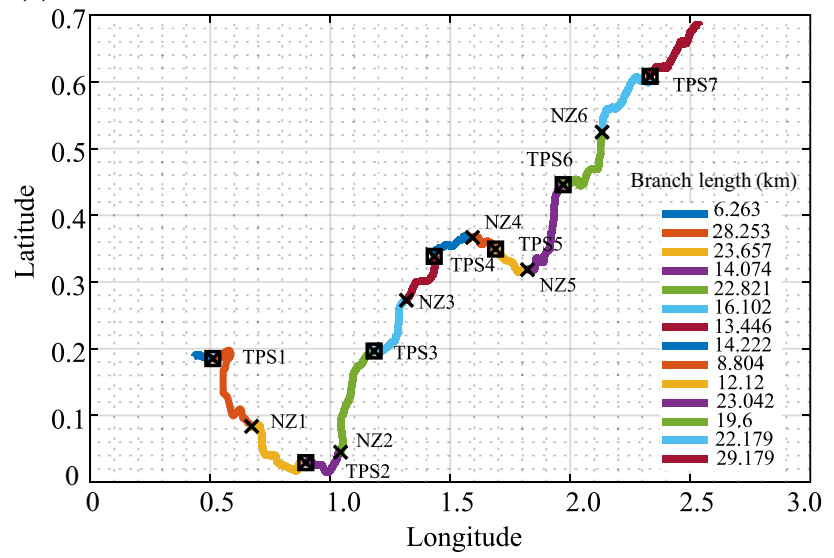

(b)

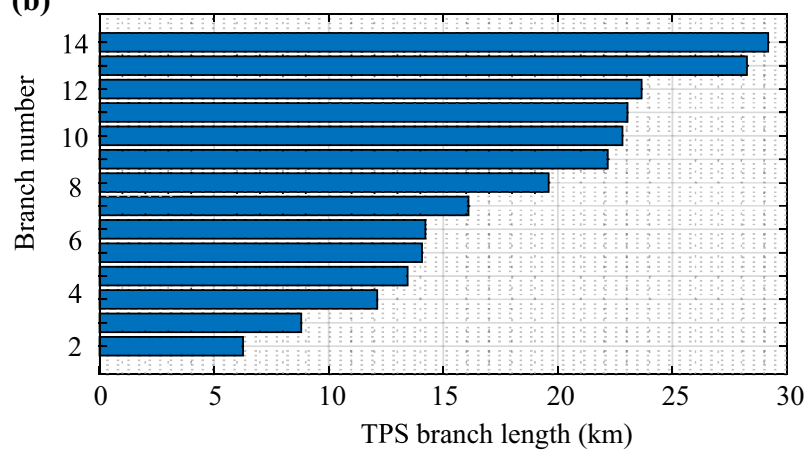

Fig. 13 a Line geographic relative coordinates (different $x$ and $y$ scales) and $\mathbf{b}$ branch lengths (in ascending order) of a $250 \mathrm{~km}$ railway line with 7 TPS

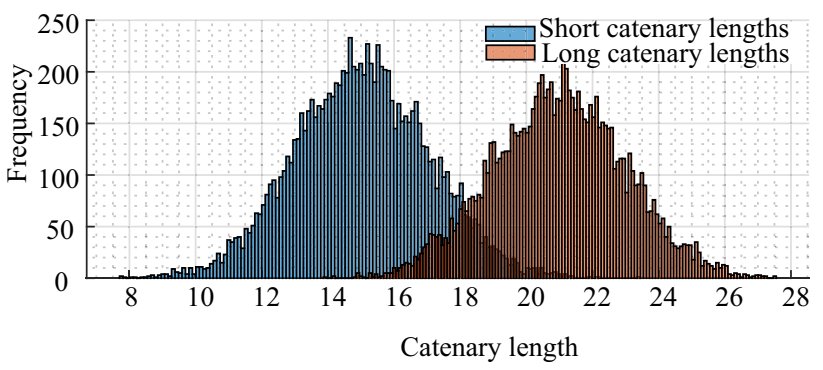

Fig. 14 Result histogram of 10000 branch lengths for the two defined clusters

The seven TPS of the $250 \mathrm{~km}$ railway line under analysis have the histogram in Fig. 15 regarding the number of trains, after the analysis of an extensive time-window concerning a specified time-table. From the results presented, two clusters of trains can be considered:

- Dense area, covered by TPS1, TPS2, TPS6 and TPS7;

- Sparse area, covered by TPS3, TPS4 and TPS5.

The two clusters are presented in the blue bars of Fig. 16. Based on the available data, the number of trains for both types of TPS follows a Poisson PDF, defined as:

- Dense area, with $\mathcal{P} o i s(\lambda=1.4)$;
- $\quad$ Sparse area, with $\mathcal{P} o i s(\lambda=0.3)$;

where the Poisson PDF is given by (6) and $\lambda$ is equal to the expected value of $k$.

$F(k, \lambda)=\frac{\mathrm{e}^{-\lambda} \lambda^{k}}{k !}$.

Then, this can be better illustrated in the fitting bar graph of Fig. 16.

Regarding the TPS power consumption, a similar analysis can also be made in Fig. 17.

Considering the two clusters of TPS (dense and sparse areas), the power consumption is also directly related to this cluster, as visible in Fig. 17.

Based on the available data, the clustered TPS power consumption can be approximated to a log-normal PDF, as follows:

- Dense area, with $\mathcal{L}$ ognormal $(\mu=\ln (5 \mathrm{e} 6), \sigma=0.75)$;

- Sparse area, with $\mathcal{L}$ ognormal $(\mu=\ln (1 \mathrm{e} 6), \sigma=1.00)$;

where the log-normal PDF is given by

$F(x,[\mu \sigma])=\frac{1}{x \sigma \sqrt{2 \pi}} \exp \left(-\frac{(\ln x-\mu)^{2}}{2 \sigma^{2}}\right)$.

The histogram of the clustered TPS power consumption and respective fitting is visible in Fig. 18.

As stated, the data of power consumption presented in Fig. 18 were fitted to the proposed log-normal PDF. However, this approximation should be taken with caution, since the performed statistical hypothesis tests from [51] do not prove that the data fits the distribution with a high degree of confidence.

Regarding the train power consumption, from the available data is visible that this parameter does not follow any PDF. Even if the authors in [41] shows a possible fitting of multiple normal PDF, the histogram results of Fig. 19 lack the fitting for specific distribution functions. Then, this work assumes a uniform PDF for the train power to comprise the diversity of types of trains present in the dataset of more than 300 train journeys.

Regarding the train power factor, from the analysis of all available data, is visible a possible dependence of the train power factor as a function of the train power, as illustrated in Fig. 20.

For lower train power consumption values, the train power factor varies from 0.4 to 1 . However, and as expected, for higher train power consumption values, the train power factor is closer to unitary.

Then, a bivariate PDF is fitted, where the power factor follows a normal distribution function and the $\mu$ and $\sigma$ values depend on the train power consumption. Specifically, as illustrated in Fig. 21, it was approximated these values to sigmoid functions. 

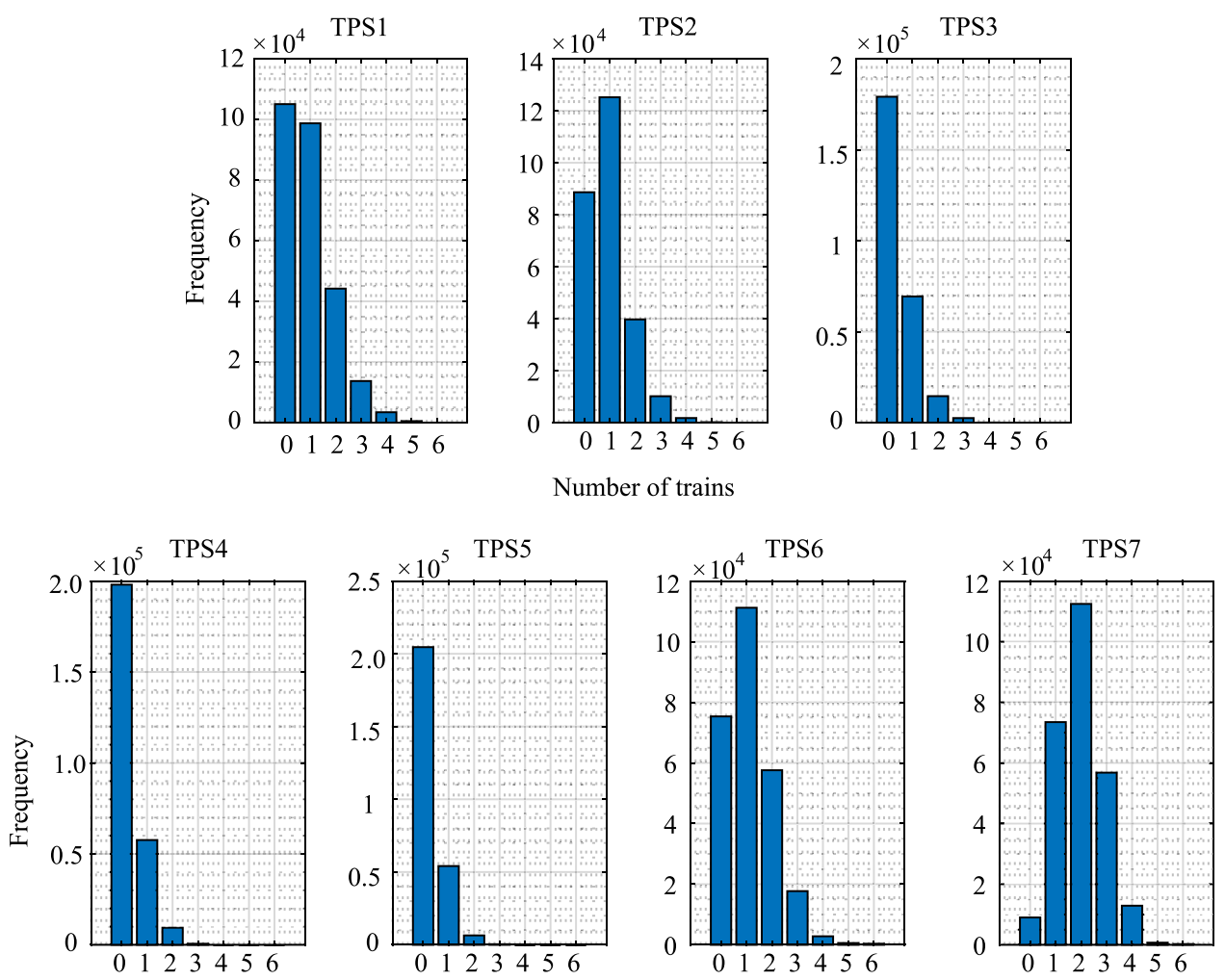

Number of trains

Fig. 15 Frequency of trains in a railway line covered by 7 TPS. The frequency corresponds to the number of seconds of a day where the TPS is feeding $t$ trains
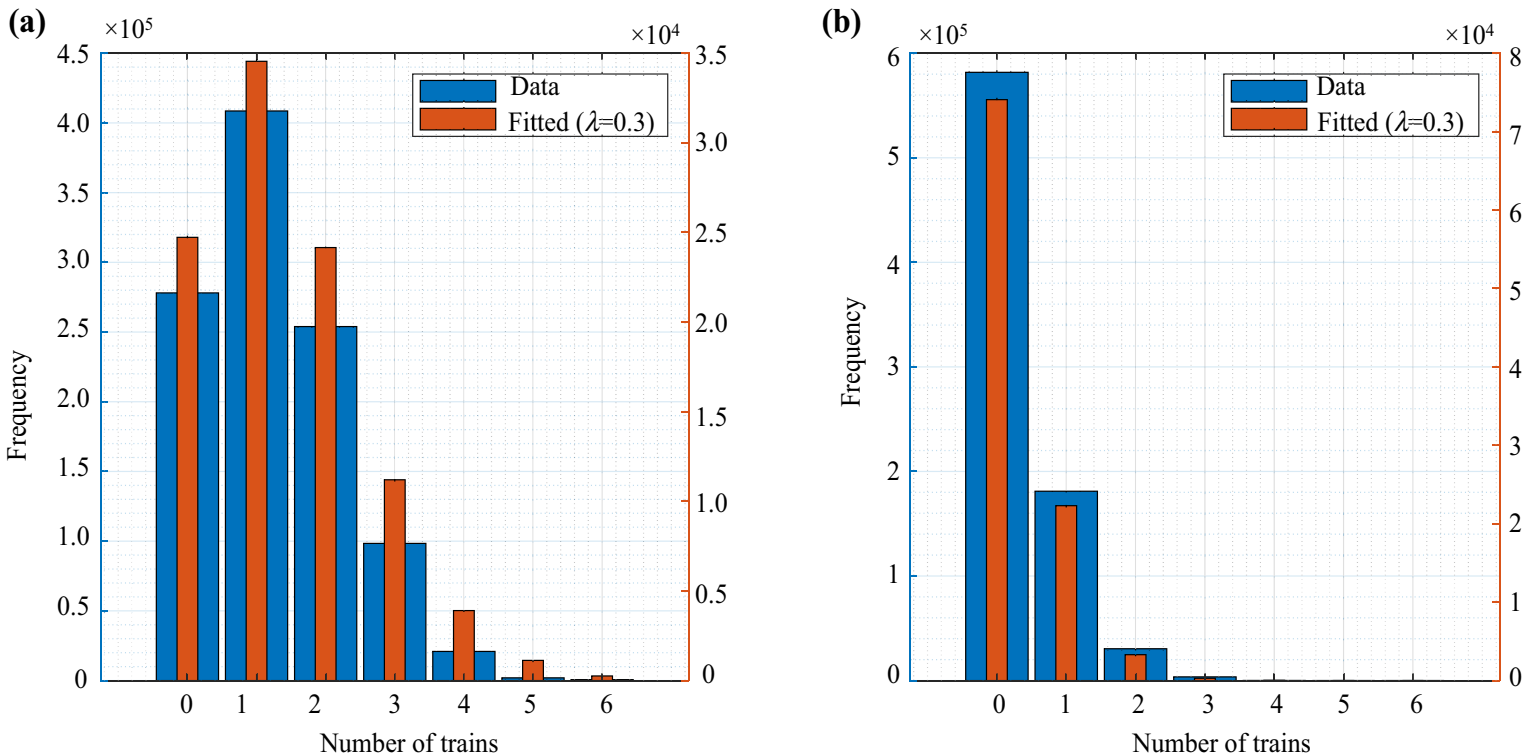

Fig. 16 Frequency of trains for the two clusters and respective fitting: a dense TPS; b sparse TPS 

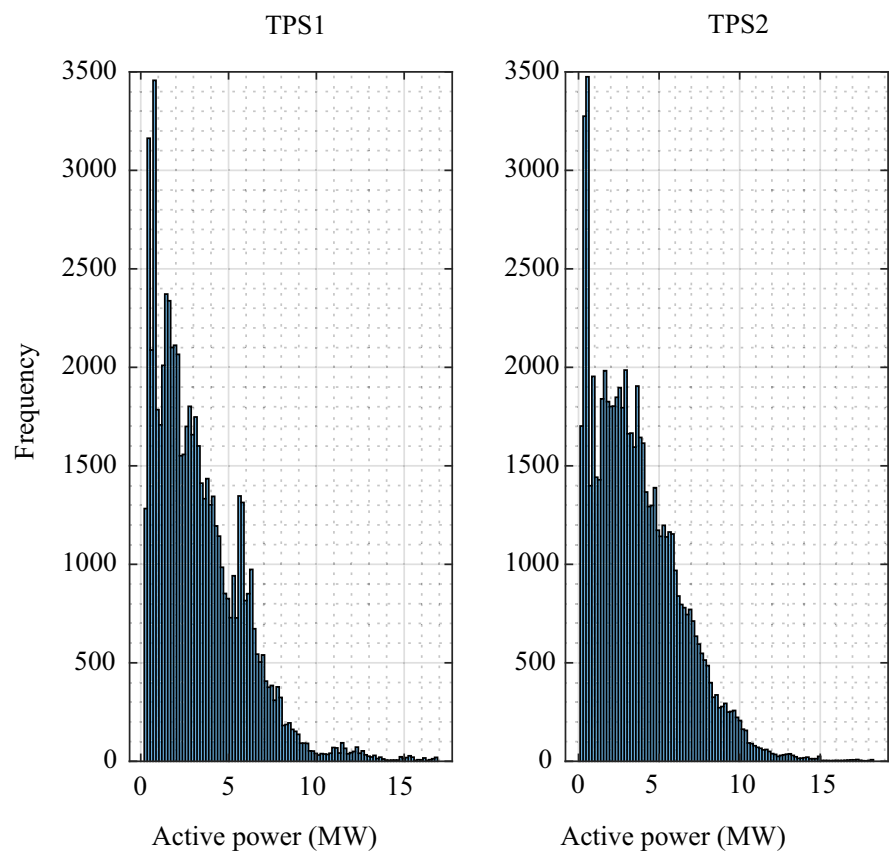

TPS3

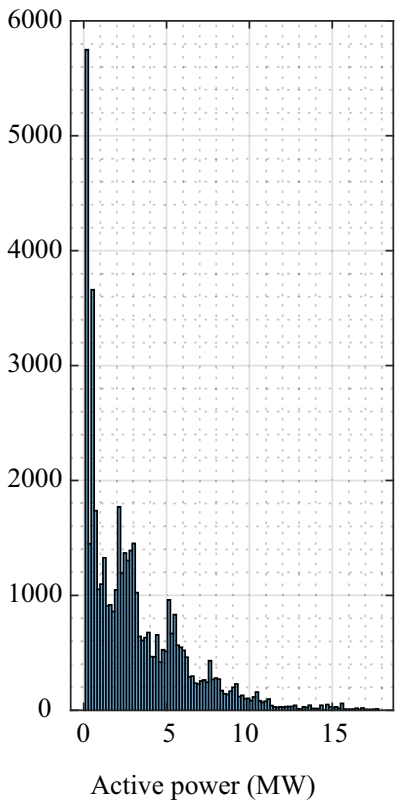

TPS4

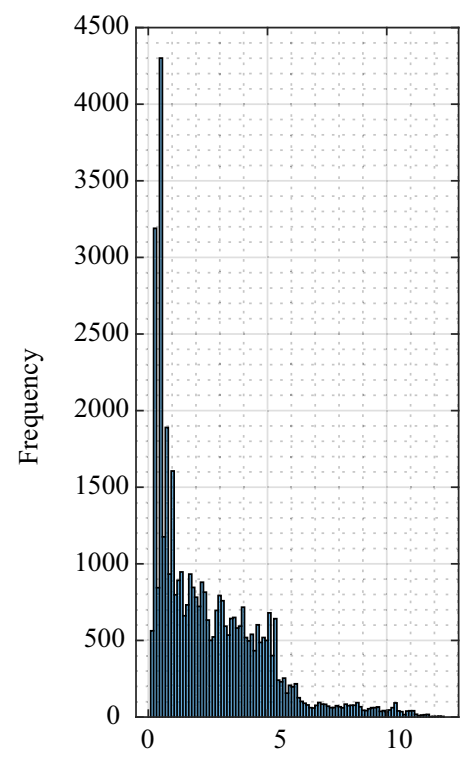

Active power (MW)
TPS5

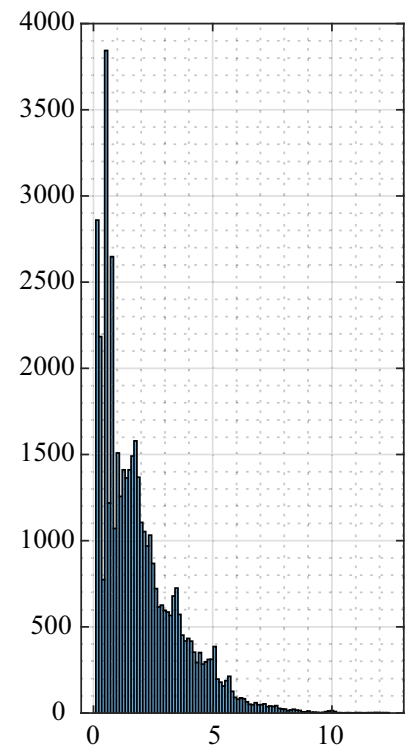

Active power (MW)

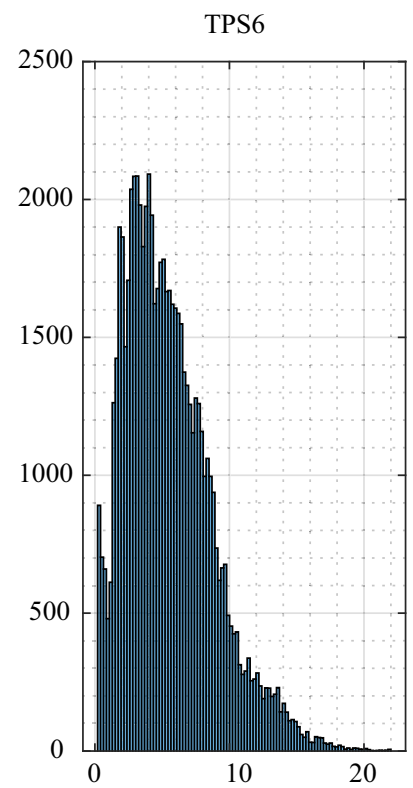

Active power (MW)
TPS7

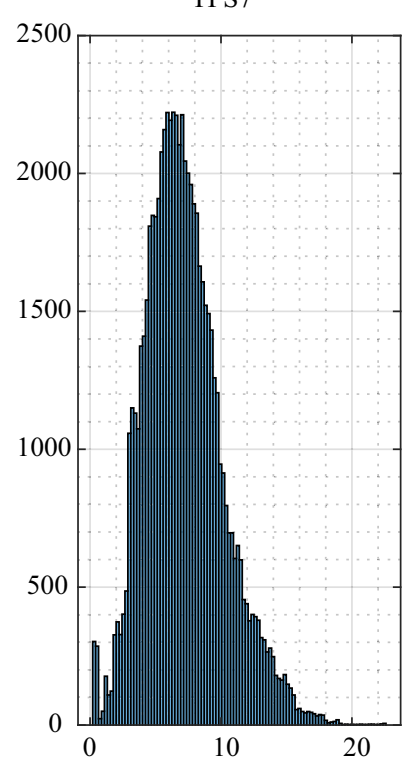

Active power (MW)

Fig. 17 Histogram graph of active power consumption for all 7 TPS of a $250 \mathrm{~km}$ railway line

The resultant bivariate surface after applying the $\mu$ and $\sigma$ curve values is presented in Fig. 22.

\subsection{Scenario generation algorithm}

The previous analysis is essential to define an automatic scenario generation that covers the universe of possible scenarios of a railway electrification line. Fig. 23 proposes an algorithm to generate a new scenario.
The first step is to generate a random length cluster, following a Bernoulli PDF for each TPS branch, resulting in four equally distributed scenarios:

- $\quad$ TPS $1 \in\{$ short, long $\}$;

- $\mathrm{TPS} 2 \in\{$ short, long $\}$.

Then, the length of the branch follows a normal PDF, as stated in the $\mu$ and $\sigma$ values that led to Fig. 14 . 

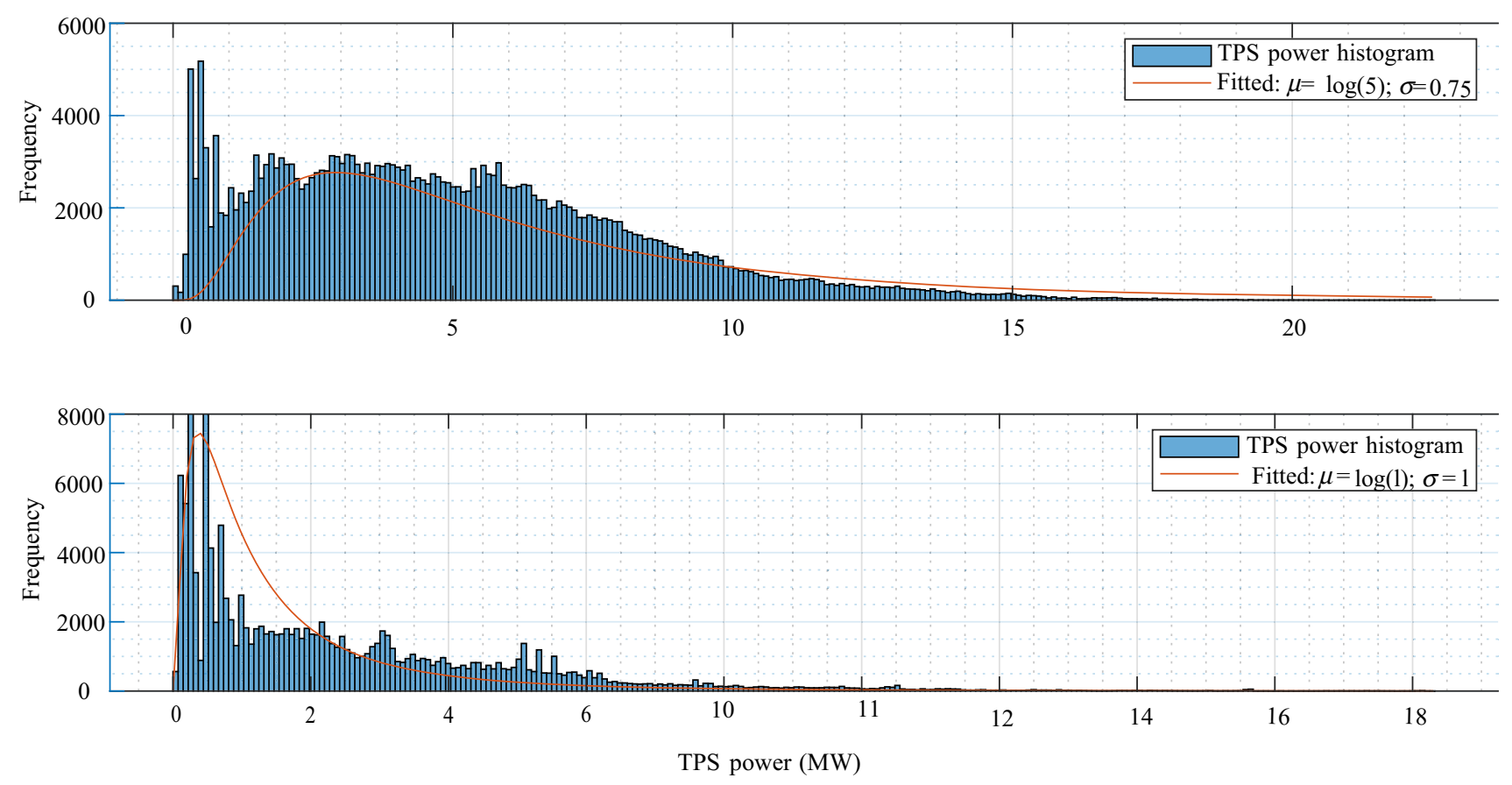

Fig. 18 Histogram graph of active power consumption for the two clusters of TPS and respective fitting: (a) dense TPS; (b) sparse TPS

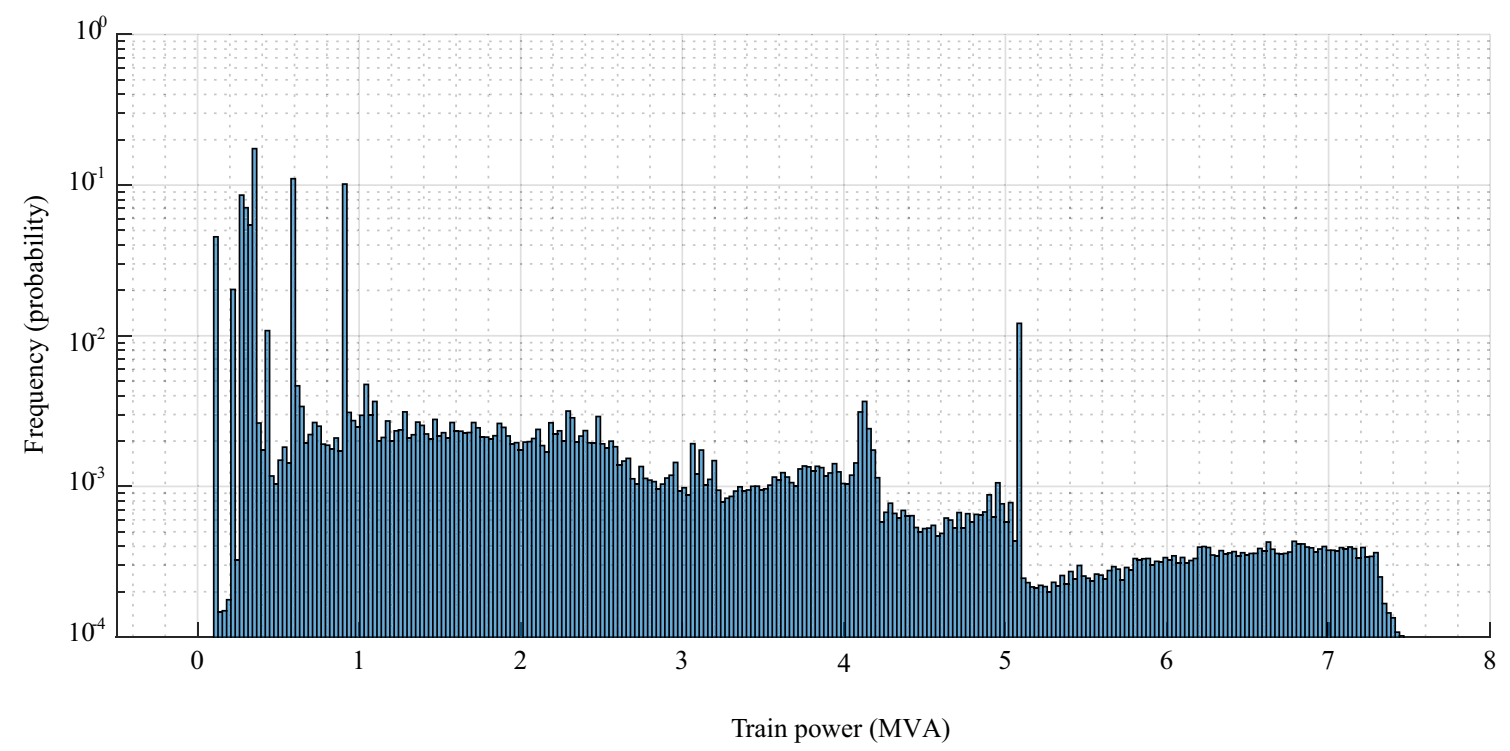

Fig. 19 Histogram graph of all train apparent power consumption. In this histogram, the frequency is in a logarithmic scale

Furthermore, the third step is to generate a random type of TPS, or TPS cluster, following a Bernoulli PDF as well, resulting in four equally distributed scenarios:

- $\quad$ TPS1 $\in\{$ sparse, dense $\}$;

- $\mathrm{TPS} 2 \in\{$ sparse, dense $\}$.

From the definition of the TPS cluster, the number of trains is generated in step four, based on the Poisson PDF that led to the results presented in Fig. 16.
The fifth step is the generation of the TPS total power consumption, which depends on the TPS cluster. Both sparse and dense TPS power consumptions follow a lognormal PDF, where the $\mu$ and $\sigma$ parameters are the ones that led to Fig. 18.

Then, this TPS power consumption is associated with the number of trains on the branch, where the power of each train is generated using a uniform PDF between a minimum and a maximum, and a final gain is applied to all 


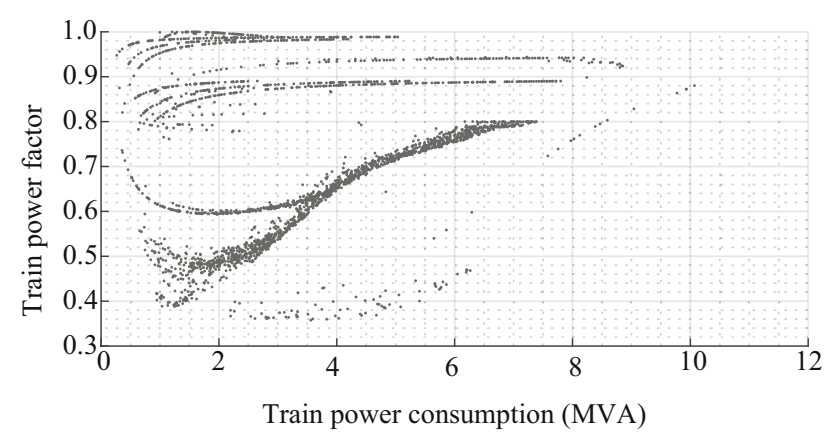

Fig. 20 Evaluation of the train power factor as function of apparent power
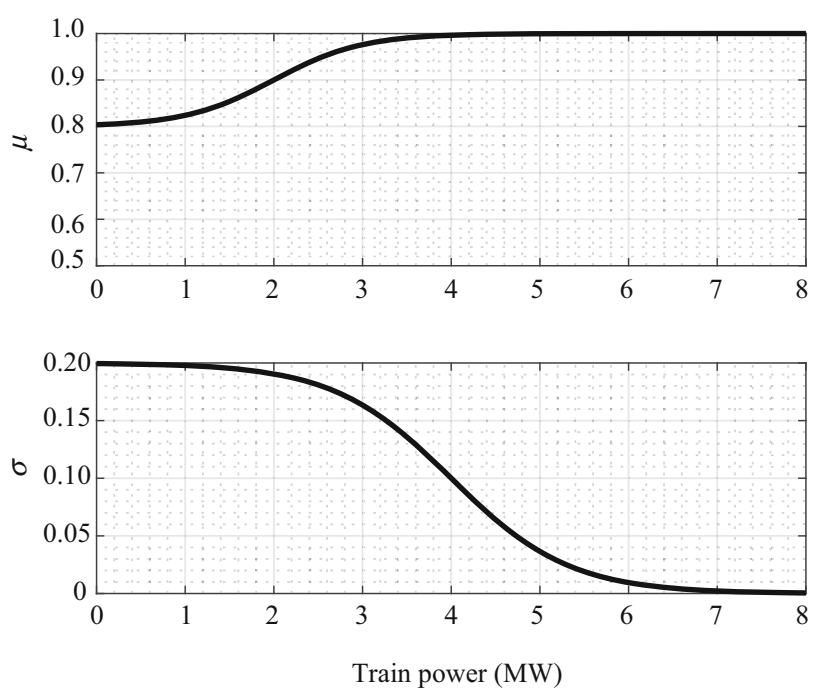

Fig. 21 Fitting of $\mu$ and $\sigma$ values of the normal PDF for the power factor as function of train power

train power consumptions to ensure that the sum of all individual powers is equal to the TPS power consumption selected in step five.

Using a normal PDF for each train, step seven generates a random power factor using the estimated train power consumption and following the $\mu$ and $\sigma$ parameters that led to the results in Figs. 21 and 22.

Finally, for each TPS branch, based on the number of trains and the TPS branch length, the position of each train is estimated using a uniform PDF (a minimum distance between trains is ensured in this phase).

\subsection{Monte Carlo metrics}

In previous subsections, all the possible parameters that affect the analysis of the inclusion of a PTD in a railway line were extensively analyzed. As stated in the previous reduced scenario section, the scenarios where the advantages (in terms of system losses) justify the adoption of a PTD are when the train is near the NZ. However, the train position follows uniform PDF. The type of conclusions to be made should be: "With the adoption of a PTD, during more than $60 \%$ of the operation time-frame, the inclusion of the PTD leads to an increase of system power losses", and not "Only if all trains are closer to NZ during the operation timewindow, is justified the adoption of the PTD”.

The Monte Carlo analysis must consider the analysis of the two parameters-the TPS length cluster and the TPS density cluster-that are fixed for a specific railway line. This analysis allows the identification of the relevant characteristics of a railway line where the study of the system power losses is essential to support this identification. The Monte Carlo analysis performed in this work will be focused on listing the combination of characteristics of a railway line (short or long TPS branches and dense or sparse TPS clusters) that contribute to a higher number of advantageous scenarios.

Regarding the number of scenarios necessary to be evaluated, let us consider a repetitive simulation of 1000 randomly generated scenarios, throughout 1000 iterations (thus a total of one million scenarios), as illustrated in Fig. 24. This figure presents the result metric for the mean value of the power loss difference (losses after minus losses before the installation of PTD).

The mean value converges to a power loss difference of $11.62 \mathrm{~kW}$ after the inclusion of a PTD. It is visible in the results of Fig. 24 that if only 1000 scenarios are considered for analysis, the average can vary from $7.33 \mathrm{~kW}$ to $16.97 \mathrm{~kW}$, which might be unacceptable, $( \pm 41.5 \%$ maximum error, when compared to the 1 million average). If there are considered 10000 scenarios, from Fig. 24, the same value can vary from 10.3 to $12.51 \mathrm{~kW}( \pm 9.4 \%$ maximum error, when compared to the 1 million average). When 100000 scenarios are considered, the maximum error is reduced to $\pm 1.72 \%$, from a value variation from 11.4 to $11.8 \mathrm{~kW}$.

Thus, only with a considerable amount of simulations is possible to increase the confidence level on the conclusions. The minimum number of simulations required and the computational needs to achieve the conclusions should be appropriately balanced. It could be argued that with this analysis, the degree of confidence of the results generated through 100000 scenarios is better than $2 \%$.

Regarding the sigma value, similar convergence conclusions can be made. Both the average and variance values are also useful to evaluate the percentage of scenarios where the inclusion of the PTD is advantageous, as illustrated in Fig. 25.

Since the PTD for the 1 million simulated scenarios is modeled without constant power losses (the PTD only has conduction power losses), there is a considerable 


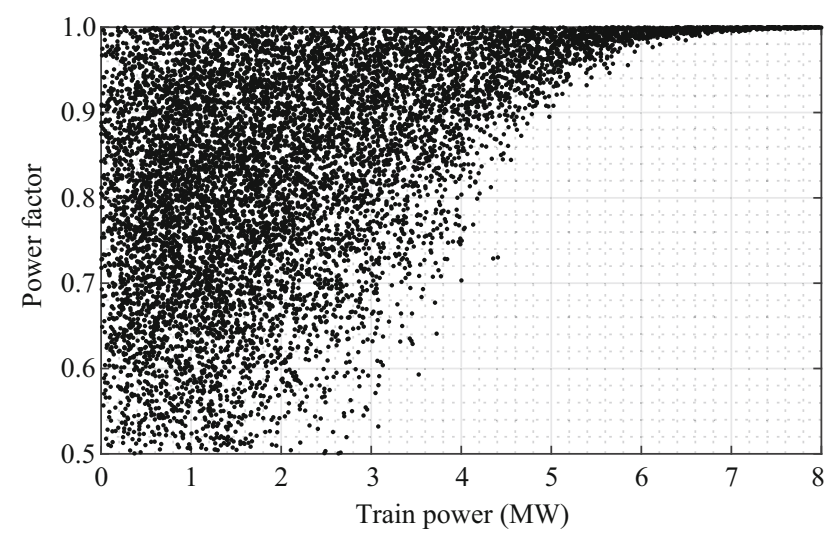

Fig. 22 Resultant randomly generated train power scenarios (active power plus power factor) for proposed variable $\mu$ and $\sigma$ values

percentage of scenarios where the PTD is advantageous in terms of system power losses.

The percentage value of the advantageous scenarios can be obtained from the definition of the cumulative distribution function for the normal PDF, following:

$\Phi(x \leq 0)=\frac{1}{2}\left[1+\operatorname{erf}\left(\frac{-\mu}{\sigma \sqrt{2}}\right)\right]$,

where $\operatorname{erf}(z)$ is the Gauss error function, given by

$\operatorname{erf}(z)=\frac{1}{\sqrt{2 \pi}} \int_{0}^{z} \mathrm{e}^{-t^{2} / 2} \mathrm{dt}$.
As example, for the given histogram of Fig. 25, the percentage value of the advantageous scenarios, $\Phi(x \leq 0)$, is $39.7 \%$.

The final results presented in the following section are obtained with the simulation of 100000 scenarios (and a different PTD).

\section{Results and discussion}

This section addresses the evaluation of the PTD inclusion, for 100000 different scenarios. In particular, the comparison is on the different clusters of TPS and branch lengths.

Two different analyses are made with different power transfer devices: i) a PTD with typical permanent power losses, with a value of $50 \mathrm{~kW}$ corresponding to $20 \%$ of the total power losses; and ii) a PTD with reduced permanent power losses, $25 \mathrm{~kW}$ corresponding to $10 \%$ of the total PTD power losses. The power losses at nominal power are the same, $2 \%$ corresponding to $250 \mathrm{~kW}$ of power losses (when the PTD is in nominal operation, transferring 12.5 MW from one section to the other).

The results for 100000 scenarios in the following subsections are presented in Tables $1-4$, where each row comprises 10000 simulated scenarios. Each cell of Tables 1-4 refer to approximately $10000 \backslash 16$ scenarios.

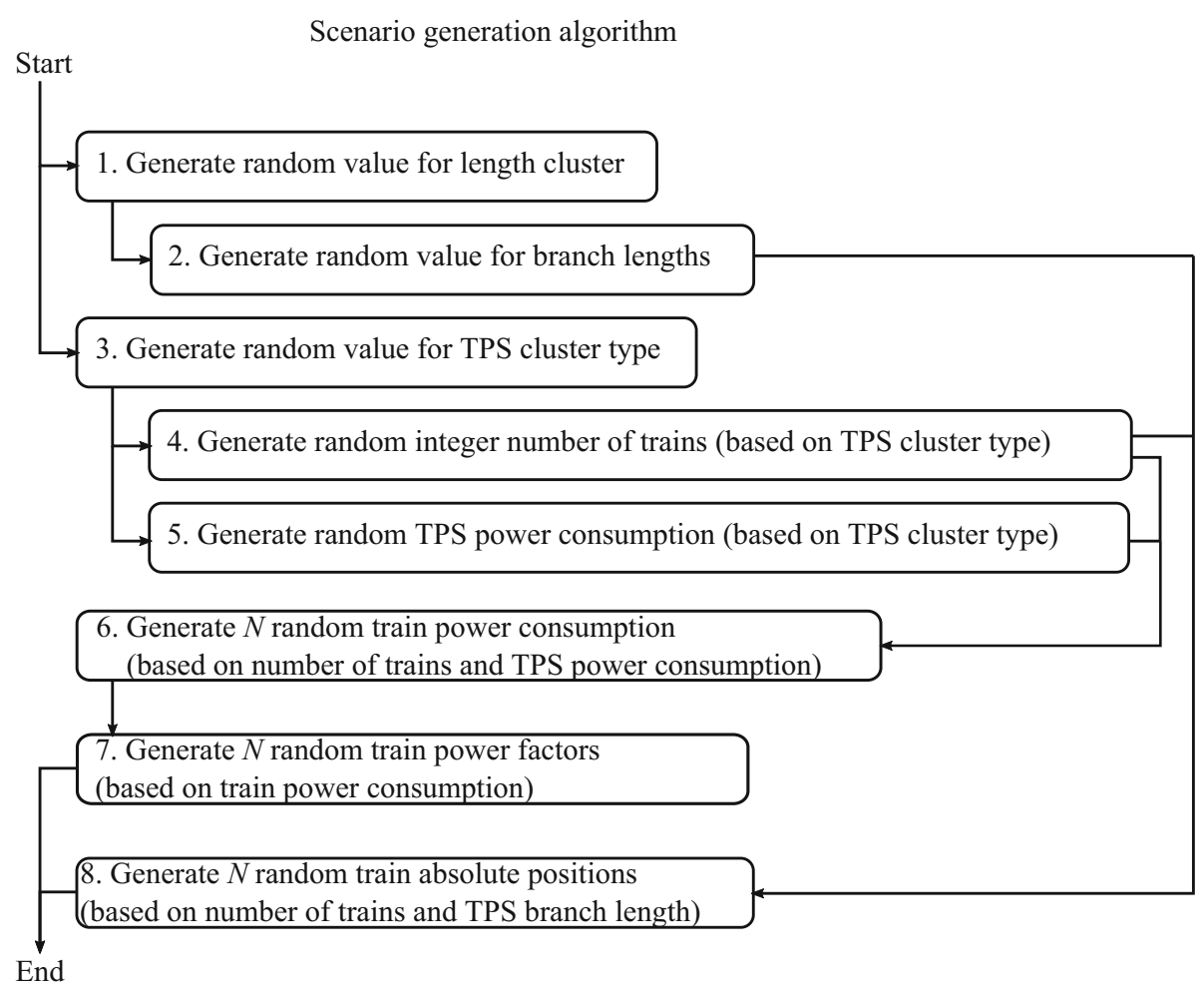

Fig. 23 Algorithm for the generation of a randomly distributed scenario 

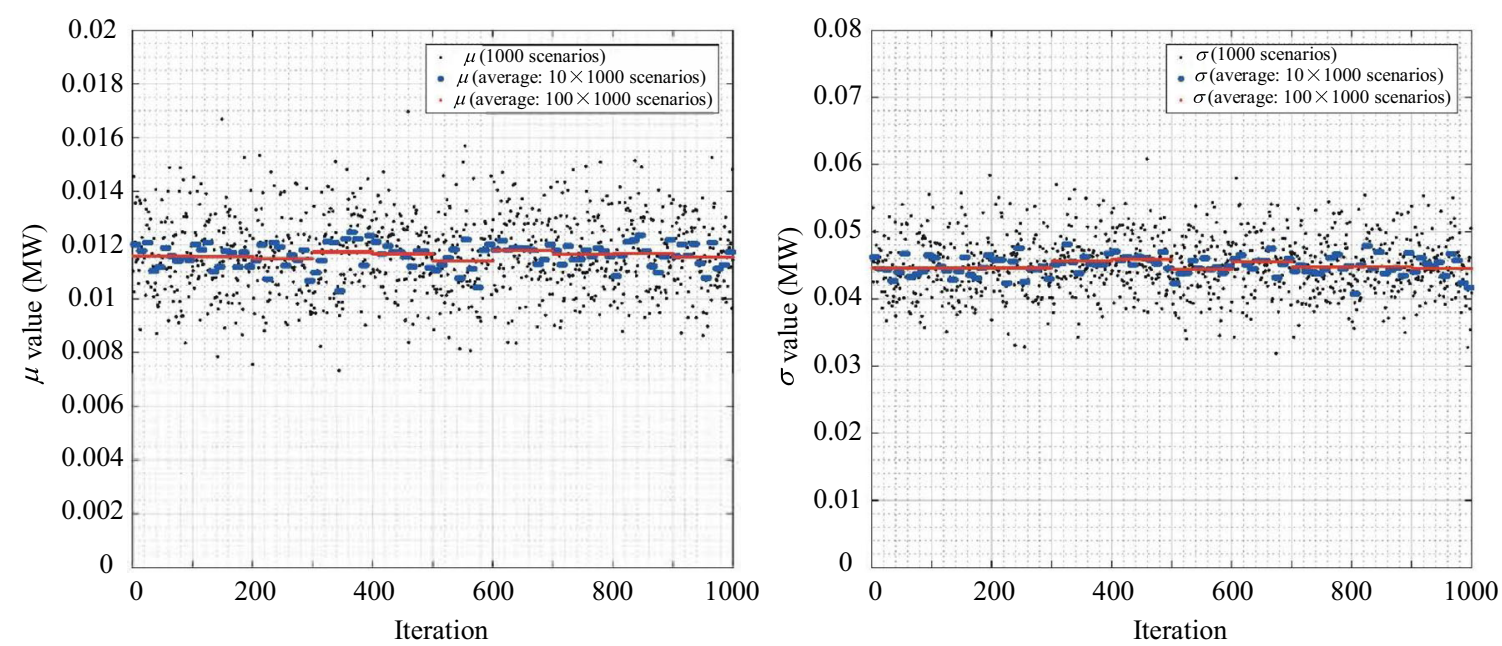

Fig. 24 Evaluation of $\mu$ and $\sigma$ values for 1 million simulated scenarios

\subsection{Power transfer device with typical power losses}

The results were obtained with a PTD with a nominal power of 12.5 MVA, where the total nominal losses are $2 \%$ and $20 \%$ of the total nominal losses are constant ones.

Table 1 presents the average of the system power losses difference. The global average value is $55.8 \mathrm{~kW}$. This means that in the average of the simulated scenarios, the inclusion of a PTD result in a power balance between two TPS with the disadvantage of increasing the losses in an average amount of $55.8 \mathrm{~kW}$. Then, from the results of Table 1, further conclusions can be obtained:
- If one TPS has a short branch and is a high density one, and the other TPS has a long branch and sparse density, then the average system power losses difference is near $10 \mathrm{~kW}$ higher than the global average (columns 6 and $11)$

- If one TPS has a long branch and high density, and the other TPS has a short branch and sparse density, then the average system power losses difference is around $6.3 \mathrm{~kW}$ lower than the global average (columns 7 and 10).

In addition to the average value, Table 2 presents the variance of the system power losses difference. Based on the application of the expression in (8) using the $\mu$ and $\sigma$

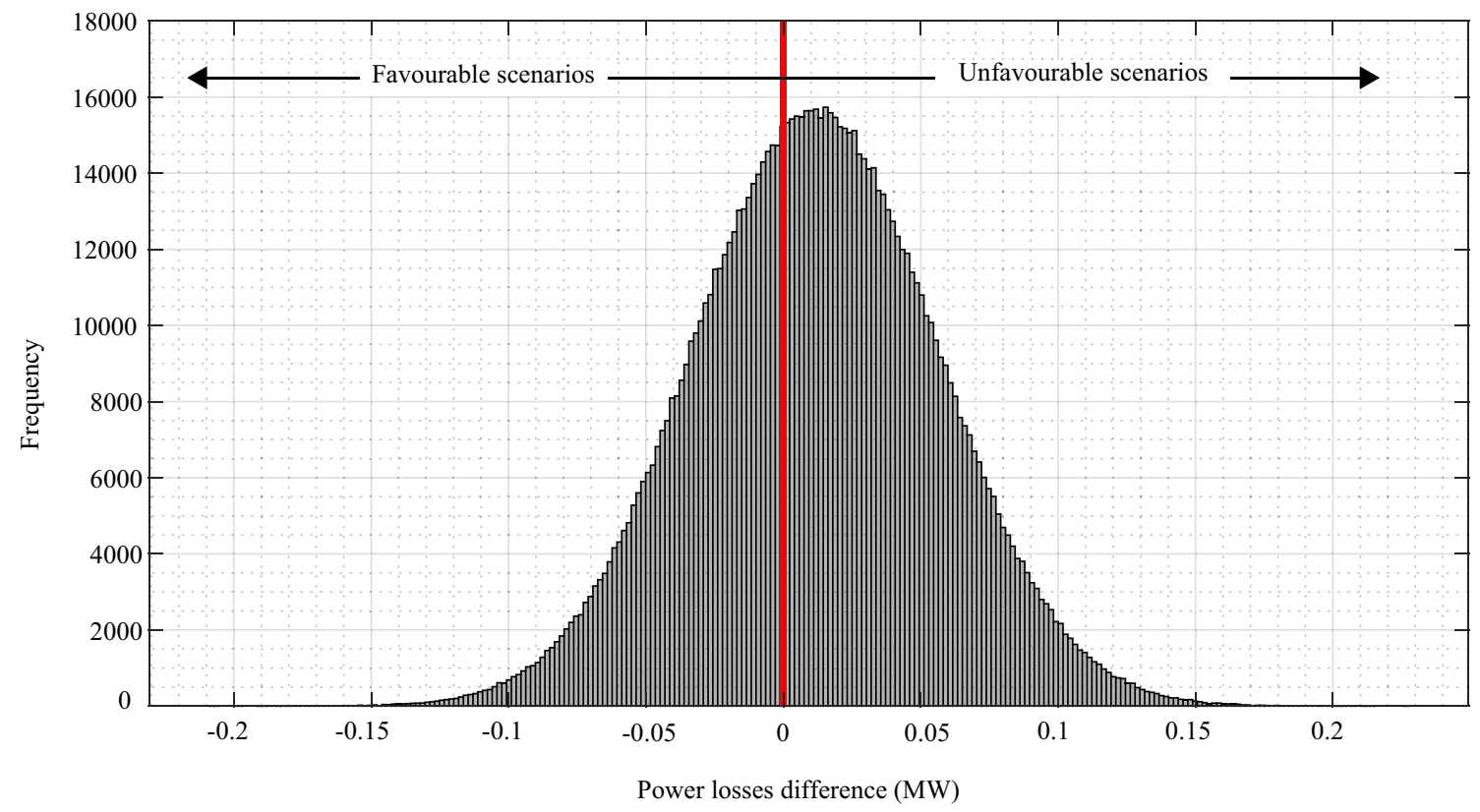

Fig. 25 Histogram of the power loss difference values for 1 million simulated scenarios 
Table 1 Evaluation of system power losses difference (after minus before, average, in $\mathrm{kW}$ ) with and without the inclusion of PTD for different clusters: TPS density and branch lengths

\begin{tabular}{|c|c|c|c|c|c|c|c|c|c|c|c|c|c|c|c|c|}
\hline TPS1 & \multicolumn{4}{|c|}{ Dense } & \multicolumn{4}{|c|}{ Dense } & \multicolumn{4}{|c|}{ Sparse } & \multicolumn{4}{|c|}{ Sparse } \\
\hline TPS2 density & \multicolumn{4}{|c|}{ Dense } & \multicolumn{4}{|c|}{ Sparse } & \multicolumn{4}{|c|}{ Dense } & \multicolumn{4}{|c|}{ Sparse } \\
\hline TPS1 length & \multicolumn{2}{|c|}{ Short } & \multicolumn{2}{|c|}{ Long } & \multicolumn{2}{|c|}{ Short } & \multicolumn{2}{|c|}{ Long } & \multicolumn{2}{|c|}{ Short } & \multicolumn{2}{|c|}{ Long } & \multicolumn{2}{|c|}{ Short } & \multicolumn{2}{|c|}{ Long } \\
\hline TPS2 length & Short & Long & Short & Long & Short & Long & Short & Long & Short & Long & Short & Long & Short & Long & Short & Long \\
\hline Scenario & 1 & 2 & 3 & 4 & 5 & 6 & 7 & 8 & 9 & 10 & 11 & 12 & 13 & 14 & 15 & 16 \\
\hline 65.9 & 57.8 & 60.0 & 59.4 & 58.1 & 58.4 & 68.2 & 49.8 & 59.2 & 56.0 & 48.6 & 64.9 & 57.8 & 50.6 & 51.1 & 51.0 & 50.7 \\
\hline 64.1 & 61.0 & 59.4 & 57.6 & 57.1 & 56.9 & 67.3 & 47.2 & 56.4 & 59.1 & 47.4 & 67.0 & 58.8 & 50.8 & 49.9 & 50.8 & 50.5 \\
\hline 62.3 & 57.5 & 57.3 & 59.3 & 62.5 & 58.5 & 64.8 & 50.3 & 56.7 & 58.2 & 48.7 & 64.4 & 58.6 & 50.6 & 50.7 & 50.6 & 51.1 \\
\hline 60.4 & 56.3 & 55.3 & 56.6 & 57.6 & 58.1 & 67.1 & 46,9 & 57.0 & 54.1 & 49.3 & 65.6 & 59.9 & 49.9 & 50.3 & 50.8 & 50.3 \\
\hline 58.6 & 56.6 & 58.4 & 57.8 & 58.3 & 56.1 & 65.8 & 48.8 & 60.7 & 56.3 & 47.3 & 66.4 & 58.1 & 50.9 & 50.6 & 50.3 & 50.2 \\
\hline 56.8 & 57.5 & 61.5 & 53.1 & 59.3 & 58.6 & 65.2 & 48.4 & 55.7 & 58.5 & 51.8 & 65.6 & 56.6 & 50.8 & 51.0 & 50.8 & 50.3 \\
\hline 55.0 & 57.3 & 55.6 & 57.1 & 56.6 & 57.1 & 66.0 & 49.7 & 56.6 & 59.4 & 49.9 & 68.0 & 56.2 & 50.3 & 50.8 & 50.1 & 50.4 \\
\hline 53.2 & 60.0 & 56.2 & 57.0 & 55,4 & 57.1 & 64.9 & 54.9 & 55.8 & 57.9 & 47.8 & 63.5 & 55.5 & 50.5 & 50.5 & 50.3 & 50.2 \\
\hline 51.4 & 56.4 & 59.1 & 55.9 & 60.2 & 60.3 & 63.7 & 49.0 & 60.0 & 58.1 & 52.4 & 66.2 & 56.1 & 50.5 & 50.8 & 50.6 & 50.5 \\
\hline 49.5 & 58.0 & 58.8 & 54.6 & 58.5 & 59.7 & 65.8 & 50.6 & 52.6 & 55.6 & 52.1 & 65.3 & 55.1 & 50.7 & 51.0 & 50.7 & 50.8 \\
\hline Average & 57.8 & 58.2 & 56.8 & 58.4 & 58.1 & 65.9 & 49.5 & 57.1 & 57.3 & 49.5 & 65.7 & 57.3 & 50.6 & 50.6 & 50.6 & 50.5 \\
\hline
\end{tabular}

Table 2 Evaluation of system power losses difference (after minus before, variance, in $\mathrm{kW}$ ) with and without the inclusion of PTD for different clusters: TPS density and branch lengths

\begin{tabular}{|c|c|c|c|c|c|c|c|c|c|c|c|c|c|c|c|c|}
\hline TPS1 & \multicolumn{4}{|c|}{ Dense } & \multicolumn{4}{|c|}{ Dense } & \multicolumn{4}{|c|}{ Sparse } & \multicolumn{4}{|c|}{ Sparse } \\
\hline TPS2 density & \multicolumn{4}{|c|}{ Dense } & \multicolumn{4}{|c|}{ Sparse } & \multicolumn{4}{|c|}{ Dense } & \multicolumn{4}{|c|}{ Sparse } \\
\hline TPS1 length & \multicolumn{2}{|c|}{ Short } & \multicolumn{2}{|c|}{ Long } & \multicolumn{2}{|c|}{ Short } & \multicolumn{2}{|c|}{ Long } & \multicolumn{2}{|c|}{ Short } & \multicolumn{2}{|c|}{ Long } & \multicolumn{2}{|c|}{ Short } & \multicolumn{2}{|c|}{ Long } \\
\hline TPS2 length & Short & Long & Short & Long & Short & Long & Short & Long & Short & Long & Short & Long & Short & Long & Short & Long \\
\hline Scenario & 1 & 2 & 3 & 4 & 5 & 6 & 7 & 8 & 9 & 10 & 11 & 12 & 13 & 14 & 15 & 16 \\
\hline 54.0 & 40.0 & 48.7 & 56.2 & 54.9 & 36.5 & 51.1 & 41.4 & 59,0 & 34.1 & 43.3 & 35.4 & 48.9 & 5.8 & 11.9 & 9.6 & 6.5 \\
\hline 48.7 & 38.1 & 55.4 & 45.8 & 51.2 & 34.4 & 42.2 & 57.1 & 40,8 & 39.9 & 46.2 & 42.1 & 53.7 & 6.2 & 6.4 & 10.2 & 6.0 \\
\hline 43.4 & 34.5 & 50.7 & 51.8 & 57.2 & 39.5 & 41.2 & 46.9 & 50,2 & 38.3 & 45.3 & 34.2 & 46.1 & 5.7 & 7.0 & 8.8 & 13.9 \\
\hline 38.1 & 36.7 & 44.1 & 49.9 & 46.0 & 29.7 & 45.4 & 46.3 & 45,2 & 28.6 & 42.5 & 41.7 & 52.2 & 8.2 & 6.5 & 8,3 & 7.0 \\
\hline 32.8 & 38.7 & 53.7 & 45.5 & 48.3 & 32.5 & 42.5 & 54.5 & 51,6 & 27.7 & 42.4 & 46.3 & 42.1 & 9.2 & 4.8 & 6.3 & 5.2 \\
\hline 27.5 & 44.4 & 71.2 & 51.9 & 57.7 & 28.9 & 42.1 & 58.3 & 46,1 & 34.7 & 41.5 & 44.6 & 46.9 & 5.7 & 13,0 & 10.5 & 6.5 \\
\hline 22.2 & 34.2 & 52.0 & 52.8 & 51.4 & 30.6 & 39.4 & 45.4 & 40,1 & 37.1 & 46.7 & 43.8 & 44.4 & 5.0 & 9.7 & 7.8 & 8.6 \\
\hline 16.9 & 38.8 & 51.0 & 41.2 & 47.6 & 32.7 & 42.7 & 47.0 & 47,4 & 31.8 & 53.8 & 39.6 & 46.5 & 5.4 & 7.1 & 7.6 & 7.3 \\
\hline 11.6 & 35.8 & 45.0 & 45.9 & 63.4 & 45.8 & 36.5 & 41.6 & 51,8 & 37.0 & 39.5 & 45.7 & 46.2 & 5.3 & 4.5 & 8.7 & 8.8 \\
\hline 6.3 & 38.6 & 65.0 & 53.8 & 62.1 & 34.4 & 46.3 & 43.9 & 45,8 & 32.8 & 44.2 & 51.3 & 48.9 & 6.6 & 8.0 & 9.5 & 7.0 \\
\hline Average & 38.0 & 53.7 & 49.5 & 54.0 & 34.5 & 43.0 & 48.2 & 47,8 & 34.2 & 44.5 & 42.5 & 47.6 & 6.3 & 7.9 & 8.7 & 7.7 \\
\hline
\end{tabular}

values from Table 1 and Table 2 , respectively, Table 3 presents the percentage of favorable scenarios where is advantageous the installation of a PTD in terms of system power losses.

From the results of Table 3 , it is identified the best scenario (column 7) where the probability of having favorable scenarios is the highest $(15.1 \%)$. This scenario is when TPS1 is dense and long and TPS2 is sparse and short. Then, more limited conclusions regarding the sparsity/density can be made, as follows:

- Due to the low $\sigma$ values in the scenarios having both sparse TPS, Table 3 shows a near-zero probability of having favorable scenarios (columns 13 to 16);
- The probability of having favorable scenarios if one of the TPS is dense is $9.1 \%$ higher than having both TPS sparse (columns 5 to 12 );

- If both TPS are dense, the probability of having favorable scenarios is $11.6 \%$ higher than having both TPS sparse (columns 1 to 4 ).

Regarding the clustering associated with the TPS lengths, other conclusions are listed:

- The probability of having favorable scenarios if both TPS branches are short is $4 \%$ (columns 1, 5, 9 and 13);

- The probability of having favorable scenarios if both TPS branches are long is $9.2 \%$ (columns 4, 8, 12 and 16); 
Table 3 Percentage of favorable scenarios $\Phi(x \leq 0)$, where the inclusion of PTD with typical power losses is beneficial for different clusters: TPS density and branch lengths

\begin{tabular}{|c|c|c|c|c|c|c|c|c|c|c|c|c|c|c|c|c|}
\hline TPS1 & \multicolumn{4}{|c|}{ Dense } & \multicolumn{4}{|c|}{ Dense } & \multicolumn{4}{|c|}{ Sparse } & \multicolumn{4}{|c|}{ Sparse } \\
\hline TPS2 density & \multicolumn{4}{|c|}{ Dense } & \multicolumn{4}{|c|}{ Sparse } & \multicolumn{4}{|c|}{ Dense } & \multicolumn{4}{|c|}{ Sparse } \\
\hline TPS1 length & \multicolumn{2}{|c|}{ Short } & \multicolumn{2}{|c|}{ Long } & \multicolumn{2}{|c|}{ Short } & \multicolumn{2}{|c|}{ Long } & \multicolumn{2}{|c|}{ Short } & \multicolumn{2}{|c|}{ Long } & \multicolumn{2}{|c|}{ Short } & \multicolumn{2}{|c|}{ Long } \\
\hline TPS2 length & Short & Long & Short & Long & Short & Long & Short & Long & Short & Long & Short & Long & Short & Long & Short & Long \\
\hline Scenario & 1 & 2 & 3 & 4 & 5 & 6 & 7 & 8 & 9 & 10 & 11 & 12 & 13 & 14 & 15 & 16 \\
\hline $15.1 \%$ & 7.5 & 10.9 & 14.5 & 14.5 & 5.5 & 9.1 & 11.5 & 15.8 & 5.0 & 13.1 & 3.3 & 11.9 & 0.0 & 0.0 & 0.0 & 0.0 \\
\hline $13.4 \%$ & 5.5 & 14.2 & 10.4 & 13.2 & 4.9 & 5.5 & 20.4 & 8.4 & 7.0 & 15.3 & 5.6 & 13.6 & 0.0 & 0.0 & 0.0 & 0.0 \\
\hline $11.7 \%$ & 4.8 & 12.9 & 12.6 & 13.7 & 7.0 & 5.8 & 14.2 & 12.9 & 6.4 & 14.1 & 3.0 & 10.2 & 0.0 & 0.0 & 0.0 & 0.0 \\
\hline $10.1 \%$ & 6.3 & 10.5 & 12.8 & 10.5 & 2.5 & 7.0 & 15.6 & 10.4 & 2.9 & 12.3 & 5.8 & 12.6 & 0.0 & 0.0 & 0.0 & 0.0 \\
\hline $8.4 \%$ & 7.2 & 13.8 & 10.2 & 11.4 & 4.2 & 6.1 & 18.5 & 12.0 & 2.1 & 13.3 & 7.6 & 8.4 & 0.0 & 0.0 & 0.0 & 0.0 \\
\hline $6.7 \%$ & 9.8 & 19.4 & 15.3 & 15.2 & 2.1 & 6.1 & 20.4 & 11.3 & 4.6 & 10.6 & 7.1 & 11.4 & 0.0 & 0.0 & 0.0 & 0.0 \\
\hline $5.0 \%$ & 4.7 & 14.3 & 14.0 & 13.5 & 3.1 & 4.7 & 13.7 & 7.9 & 5.5 & 14.3 & 6.0 & 10.3 & 0.0 & 0.0 & 0.0 & 0.0 \\
\hline $3.4 \%$ & 6.1 & 13.6 & 8.3 & 12.2 & 4.0 & 6.4 & 12.1 & 12.0 & 3.4 & 18.7 & 5.4 & 11.6 & 0.0 & 0.0 & 0.0 & 0.0 \\
\hline $1.7 \%$ & 5.8 & 9.5 & 11.2 & 17.1 & 9.4 & 4.0 & 11.9 & 12.3 & 5.8 & 9.2 & 7.4 & 11.2 & 0.0 & 0.0 & 0.0 & 0.0 \\
\hline $0.0 \%$ & 6.6 & 18.3 & 15.5 & 17.3 & 4.1 & 7.8 & 12.5 & 12.5 & 4.5 & 11.9 & 10.1 & 13.0 & 0.0 & 0.0 & 0.0 & 0.0 \\
\hline Average & 6.4 & 13.7 & 12.5 & 13.9 & 4.7 & 6.3 & 15.1 & 11.5 & 4.7 & 13.3 & 6.1 & 11.4 & 0.0 & 0.0 & 0.0 & 0.0 \\
\hline
\end{tabular}

Table 4 Percentage of favorable scenarios $\Phi(x \leq 0)$, where the inclusion of high-efficiency PTD with reduced constant power losses is beneficial for different clusters: TPS density and branch lengths

\begin{tabular}{|c|c|c|c|c|c|c|c|c|c|c|c|c|c|c|c|c|}
\hline TPS1 & \multicolumn{4}{|c|}{ Dense } & \multicolumn{4}{|c|}{ Dense } & \multicolumn{4}{|c|}{ Sparse } & \multicolumn{4}{|c|}{ Sparse } \\
\hline TPS2 density & \multicolumn{4}{|c|}{ Dense } & \multicolumn{4}{|c|}{ Sparse } & \multicolumn{4}{|c|}{ Dense } & \multicolumn{4}{|c|}{ Sparse } \\
\hline TPS1 length & \multicolumn{2}{|c|}{ Short } & \multicolumn{2}{|c|}{ Long } & \multicolumn{2}{|c|}{ Short } & \multicolumn{2}{|c|}{ Long } & \multicolumn{2}{|c|}{ Short } & \multicolumn{2}{|c|}{ Long } & \multicolumn{2}{|c|}{ Short } & \multicolumn{2}{|c|}{ Long } \\
\hline TPS2 length & Short & Long & Short & Long & Short & Long & Short & Long & Short & Long & Short & Long & Short & Long & Short & Long \\
\hline Scenario & 1 & 2 & 3 & 4 & 5 & 6 & 7 & 8 & 9 & 10 & 11 & 12 & 13 & 14 & 15 & 16 \\
\hline $24.2 \%$ & 19.1 & 22.6 & 23.5 & 24.2 & 18.1 & 21.6 & 21.8 & 25.3 & 16.6 & 19.7 & 15.1 & 23.1 & 0.0 & 2.8 & 1.3 & 0.3 \\
\hline $21.5 \%$ & 17.4 & 22.3 & 21.7 & 22.2 & 15.6 & 17.4 & 26.6 & 19.9 & 19.1 & 23.5 & 17.7 & 23.6 & 0.0 & 0.0 & 0.6 & 0.0 \\
\hline $18.8 \%$ & 19.1 & 22.6 & 23.5 & 24.1 & 18.1 & 21.6 & 21.1 & 25.3 & 16.9 & 20.9 & 15.0 & 23.1 & 0.0 & 2.8 & 1.0 & 0.3 \\
\hline $16.2 \%$ & 17.5 & 22.2 & 21.2 & 22.3 & 15.8 & 17.4 & 27.0 & 19.9 & 19.0 & 22.0 & 17.8 & 23.7 & 0.0 & 0.0 & 0.8 & 0.0 \\
\hline $13.5 \%$ & 16.5 & 22.5 & 21.6 & 22.9 & 18.2 & 17.0 & 22.4 & 22.3 & 19.1 & 22.8 & 14.6 & $20, .7$ & 0.0 & 0.1 & 0.6 & 5.7 \\
\hline $10.8 \%$ & 18.2 & 19.6 & 23.0 & 20.8 & 15.5 & 18.5 & 24.2 & 22.2 & 15.1 & 22.8 & 18.3 & 22.1 & 0.1 & 0.0 & 0.3 & 0.0 \\
\hline $8.1 \%$ & 17.8 & 23.9 & 21.3 & 22.2 & 15.0 & 18.6 & 25.0 & 21.6 & 12.8 & 23.1 & 20.0 & 21.4 & 1.0 & 0.0 & 0.0 & 0.0 \\
\hline $5.5 \%$ & 19.4 & 26.5 & 24.4 & 24.5 & 13.4 & 18.3 & 28.0 & 21.5 & 17.9 & $21, .4$ & 19.0 & 20.1 & 0.0 & 4.3 & 1.8 & 0.0 \\
\hline $2.8 \%$ & 15.8 & 22.8 & 24.7 & 23.3 & 16.0 & 15.8 & 23.5 & 17.9 & 18.0 & 22.9 & 17.1 & 20.8 & 0.0 & 1.4 & 0.1 & 0.3 \\
\hline $0.1 \%$ & 18.2 & 23.8 & 19.5 & 21.1 & 15.9 & 19.0 & 22.2 & 21.7 & 16.8 & 27.3 & 17.8 & 20.7 & 0.0 & 0.0 & 0. & 0.1 \\
\hline Average & 17.9 & 22.9 & 22.4 & 22.8 & 16.1 & 18.5 & 24.2 & 21.8 & 17.1 & 22.6 & 17.2 & 21.9 & 0.1 & 1.1 & 0.7 & 0.7 \\
\hline
\end{tabular}

- If both TPS are dense and if one TPS branch is different from the other, the probability of having favorable scenarios is $13.1 \%$ (columns 2 and 3);

- If one TPS is dense and short, and the other TPS is sparse and long (columns 6 and 11), the probability of having favorable scenarios is $6.2 \%$;

- If one TPS is dense and long, and the other TPS is sparse and short (columns 7 and 10), the probability of having favorable scenarios is $14.2 \%$.

\subsection{High-efficiency power transfer device}

A second analysis was made with a more efficient PTD. The total nominal losses are still $2 \%$ of the nominal power of $12.5 \mathrm{MW}$. The constant power losses are $10 \%$ of the nominal power losses.

Table 4 presents the percentage of favorable scenarios where is advantageous the installation of a PTD.

Similar to the analysis made for the PTD with typical power losses, the percentage results in Table 4 show the best scenario being the same (column 7), where the 
probability of having favorable scenarios is the highest $(24.2 \%)$.

The conclusions for the sparsity/density are the following:

- The scenarios having both sparse TPS show an average of $0.65 \%$ in the probability of having favorable scenarios (columns 13 to 16 );

- The probability of having favorable scenarios if one of TPS is dense is $19.3 \%$ higher than having both TPS sparse (columns 5 to 12 );

- If both TPS are dense, the probability of having favorable scenarios is $20.8 \%$ higher than having both TPS sparse (columns 1 to 4 ).

The conclusions for the clustering associated with the TPS lengths are the following:

- The probability of having favorable scenarios if both TPS branches are short is $12.8 \%$ (columns 1, 5, 9 and 13);

- The probability of having favorable scenarios if both TPS branches are long is $16.8 \%$ (columns 4, 8, 12 and $16)$;

- If both TPS are dense and if one TPS branch is different from the other, the probability of having favorable scenarios is $22.7 \%$ (columns 2 and 3 );

- If one TPS is dense and short, and the other TPS is sparse and long (columns 6 and 11), the probability of having favorable scenarios is $17.9 \%$;

- If one TPS is dense and long, and the other TPS is sparse and short (columns 7 and 10), the probability of having favorable scenarios is $23.4 \%$.

When compared to a PTD with typical constant power losses, Table 4 shows a considerable increase in all the probabilities for having favorable scenarios, as expected. This increase is on average 10.5 percentage points (scenarios 1 to 12 , with 1.2 percentage points in standard deviation).

Since the constant power losses of this PTD analysis are $25 \mathrm{~kW}$ (half of the typical PTD), if both TPS are sparse (columns 13 to 16), there is a slight increase in the probability of having favorable scenarios (in the neighborhood of $1 \%)$.

\subsection{Discussion}

The analysis previously made is useful if the study of the implementation of a PTD in the NZ is strictly subject to the system power losses. If is needed an analysis on the best location to install one PTD from a universe of possible alternatives, then the first analysis is to evaluate the branch lengths and the TPS density. In particular, when compared to all possible scenarios, it is concluded that the best scenarios are the ones where one TPS is dense and long, and the other TPS is sparse and short (columns 7 and 10) to achieve the highest probability of having favorable scenarios (14.2\% for PTD with typical constant losses, or $23.4 \%$ for highly efficient PTD).

In an opposite situation, if both TPS are sparse, from the results achieved, it is expected that the probability of having favorable scenarios is residual (near-zero for the typical PTD, and near $1 \%$ for PTD with reduced losses), leading to the conclusion that, if there is a choice, it is not justified the installation of a PTD in this cluster of TPS. As visible in Fig. 16, for this scenario, the probability of not having any train in both TPS can be estimated using the Poisson PDF for the sparse area. This value is around $54 \%$, wherein in all operation scenarios, the PTD only accounts for the unfavorable constant losses. Also, in this scenario, the probability of having one train either in one of the TPS branches is around $33 \%$, and considering Fig. 10 of the atomic scenario, from all of these scenarios, only $40 \%$ of these are advantageous.

It should be highlighted that the inclusion of the PTD installation in the railway electrification system leads always to a more balanced railway power supply. Despite the major focus of the results presented in this paper are on the system power losses, it is clear that once this system is installed, the power demand of the trains in a catenary branch is distributed among the adjacent TPS.

The compensation strategy illustrated in this work depends on the measurement of the power consumption in both TPS. Then, in a practical implementation of this strategy, it might be required a real-time communication system to transfer the measurements remotely between both TPS and the PTD control system.

Finally, this work does not address the control of the PTD reactive power. As stated in [3], a reactive power compensation device can improve the power quality in the railway electrification, specifically with the stabilization of the catenary voltage (allowing to an increase in the infrastructure capacity). In the limit scenario presented in [3], the catenary power losses can be reduced by more than $50 \%$ if the reactive power in the railway electrification is handled. The practical implementation of the strategy proposed in this work must also consider the management of the reactive power, for improved operation of the PTD system.

\section{Conclusion}

This paper addresses the effects of the inclusion of a Power Transfer Device between two sections of a railway line based on a Monte Carlo statistical analysis. The main analyzed parameters are the active power balancing in the 
two substations and the system power losses difference with/without the installation of the PTD.

The proposed methodology considers a generic electrification where several scenarios can be simulated and where each scenario has several different parameters.

From a reduced analysis scenario, the adopted strategy is capable to balance the power in both TPS with a small maximum error (5.5 \% in the presented analysis). However, this TPS power balance is achieved with non-negligible system power losses. Specifically, if it is considered an ideal TPS and an ideal PTD $\left(R_{\mathrm{tps}}=0\right.$ and $R_{\mathrm{ptd}}=0$, resulting in $\alpha_{0}=0.5$ ), if the train is in the half of the branch closer to the TPS, then the adoption of a PTD results in higher losses than if no PTD is installed.

To contemplate a generic scenario coverage, then an extensive statistical analysis was made in this paper, using data and measurements obtained from a $250 \mathrm{~km}$ railway line with 7 TPS. It was concluded that some of the parameters follow specific probability distribution functions. Specifically, the number of trains follows a Poisson PDF, the TPS power consumption follows a LogNormal PDF and the train power factor is statistically dependent on the train active power.

The final results of the Monte Carlo analysis show that, depending on the density of trains and on the branch lengths, the scenarios where these factors are different for the two TPS can result in considerable higher losses if not carefully considered. However, the PTD system can always balance the two substations, thus implementing double-side feeding and can also reduce the system total losses in some specific scenarios.

\section{Appendix}

The graphical user interfaces for the developed framework are shown in Figs. 26 and 27.

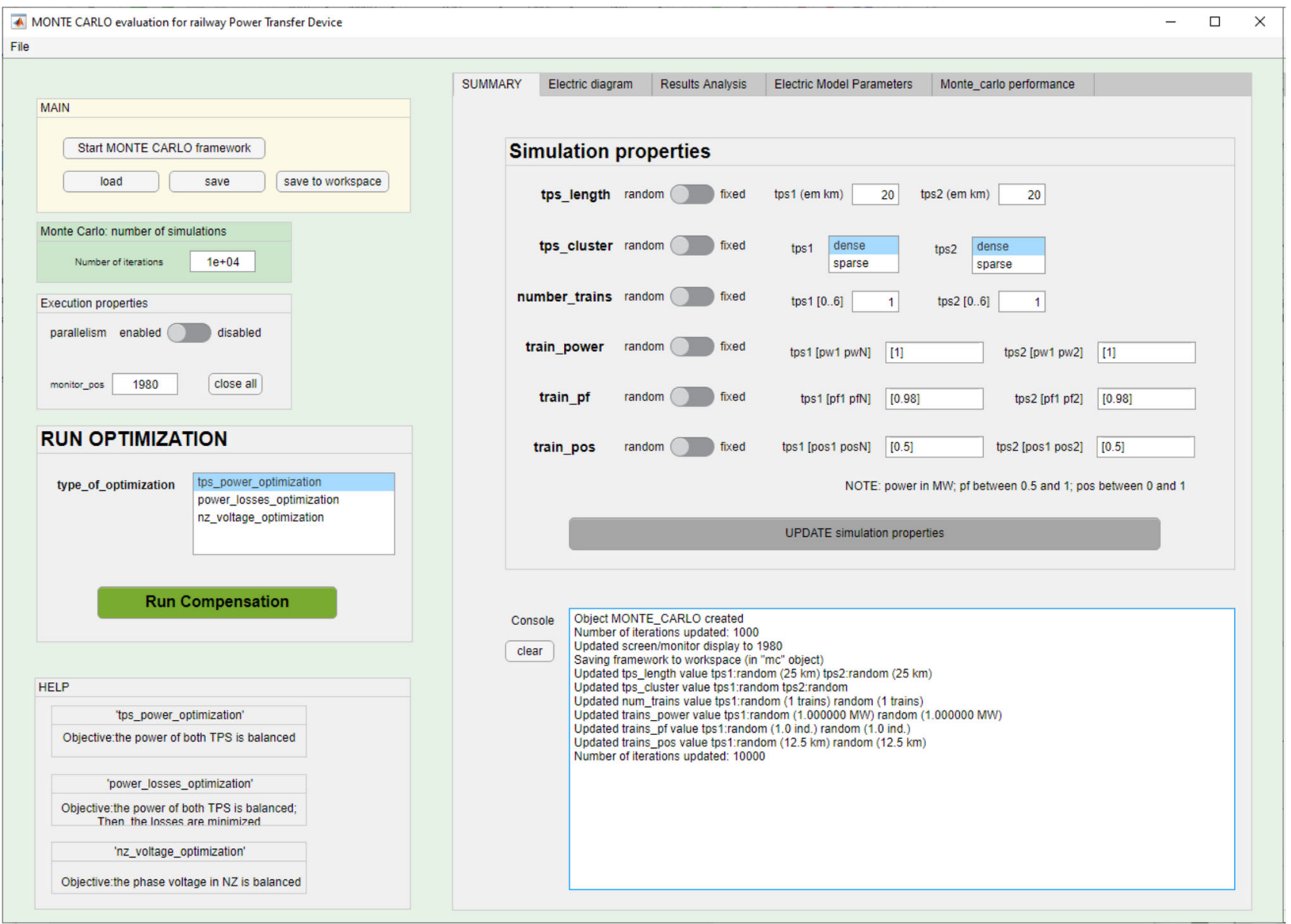

Fig. 26 Graphical user interface of the developed framework: random scenario generation menu 


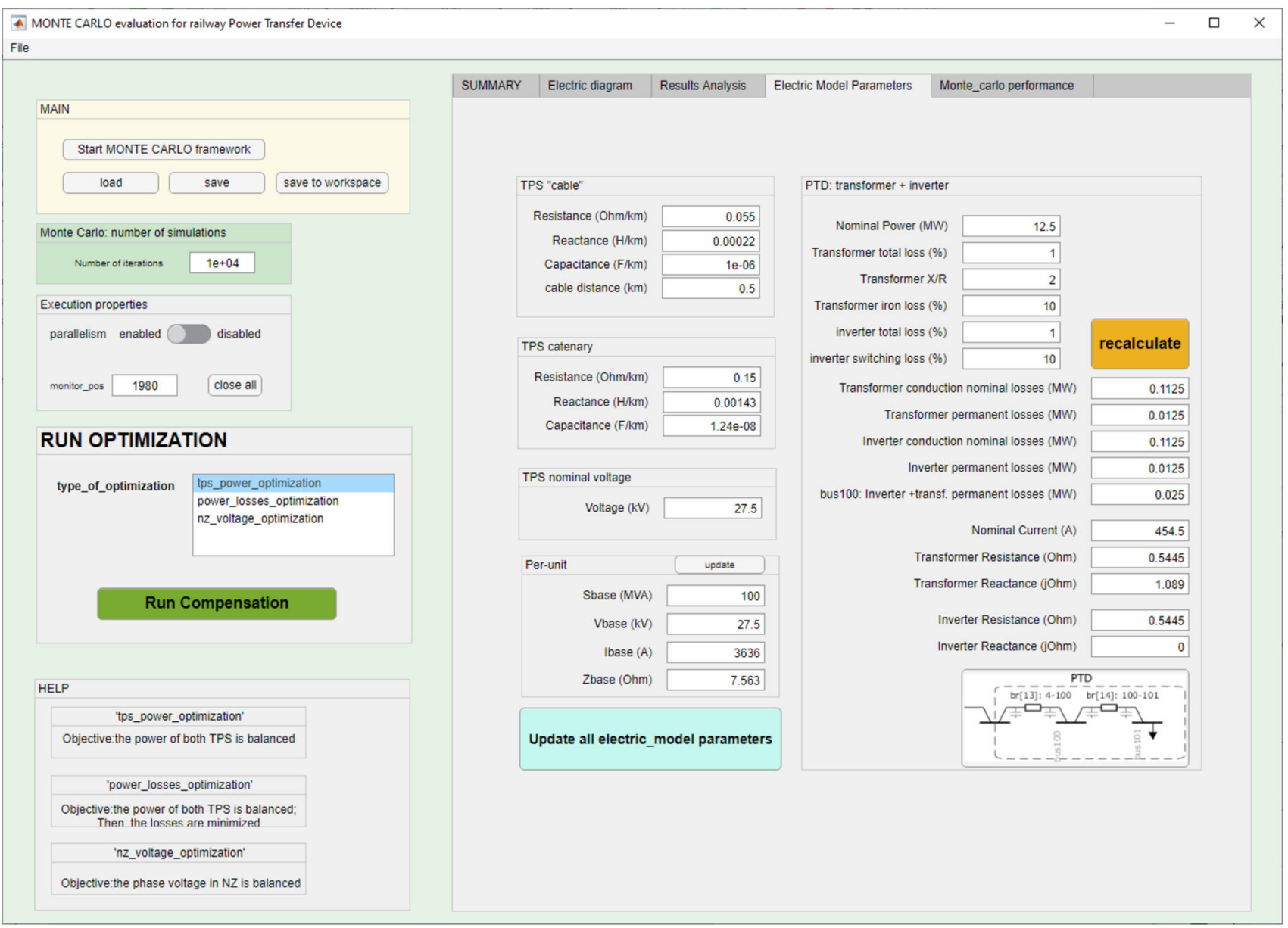

Fig. 27 Graphical user interface of the developed framework: definition of electric parameters for the catenary electrification and the PTD

Acknowledgements This research was funded by FCT (Fun- daçāo Ciência e Tecnologia) under grant PD/BD/128051/2016. The research is also associated with the Shift2Rail In2Stempo project (grant 777515). This work was partially supported by FCT R\&D Unit SYSTEC-POCI-01-0145-FEDER-006933/SYSTEC funded by FEDER funds through COMPETE2020 and by national funds through the FCT/MEC, and co-funded by FEDER, in the scope of the PT2020 Partnership Agreement.

Open Access This article is licensed under a Creative Commons Attribution 4.0 International License, which permits use, sharing, adaptation, distribution and reproduction in any medium or format, as long as you give appropriate credit to the original author(s) and the source, provide a link to the Creative Commons licence, and indicate if changes were made. The images or other third party material in this article are included in the article's Creative Commons licence, unless indicated otherwise in a credit line to the material. If material is not included in the article's Creative Commons licence and your intended use is not permitted by statutory regulation or exceeds the permitted use, you will need to obtain permission directly from the copyright holder. To view a copy of this licence, visit http://creativecommons. org/licenses/by/4.0/.

\section{References}

1. International Energy Agency (IEA) and International Union of Railways (2017) Energy consumption and CO2 emissions focus on passenger rail services.

2. Pilo E, Mazumder S, Franco I (2014) Railway electrical smart grids: an introduction to next-generation railway power systems and their operation. IEEE Electr Mag 2(3):49-55

3. Morais VA, Afonso JL, Carvalho AS, Martins AP (2020) New reactive power compensation strategies for railway infrastructure capacity increasing. Energies 13(17):4379

4. Shift2Rail Joint Undertaking (2019) Multi-Anual action plan. (amended version: 2019). Tech. rep., Luxembourg

5. Tumilowicz A, Sugarman M (2020) IN2STEMPO: How smart maintenance could help support the decarbonisation of our rail network. Global Railway Review 2020(5). https://www. globalrailwayreview.com/article/111634/global-railway-reviewissue-5-2020/

6. Tumilowicz A (2021) In2stempo - brochure: Innovative solutions in future stations, energy metering \& power supply. https:// projects.shift2rail.org/download.aspx?id=cb167b43-c2a6-4a8abd6f-41cd162a964c

7. Mariscotti A (2012) Measuring and analyzing power quality in electric traction systems. Int $\mathbf{J}$ Meas Technol Instrum Eng 2(4):21-42 
8. Pilo E, Mazumder SK, Gonzalez-Franco I (2014) Smart electrical infrastructure for AC-fed railways with neutral zones. IEEE Trans Intell Transp Syst 16(2):1-11

9. He Z, Zheng Z, Hu H (2016) Power quality in high-speed railway systems. Int J Rail Transp 4(2):71-97

10. Kaleybar HJ, Brenna M, Foiadelli F, Fazel SS, Zaninelli D (2020) Power quality phenomena in electric railway power supply systems: an exhaustive framework and classification. Energies 13(24):6662

11. Li Q (2015) New generation traction power supply system and its key technologies for electrified railways. J Mod Transp 23(1):1-11

12. Krastev I, Tricoli P, Hillmansen S, Chen M (2016) Future of electric railways: advanced electrification systems with static converters for AC railways. IEEE Electr Mag 4(3):6-14

13. Hayashiya H, Kondo K (2020) Recent trends in power electronics applications as solutions in electric railways. IEEJ Trans Electr Electr Eng 15(5):632-645

14. Bernet S (2000) Recent developments of high power converters for industry and traction applications. IEEE Trans Power Electr 15(6):1102-1117

15. Zhao C, Dujic D, Mester A, Steinke JK, Weiss M, LewdeniSchmid S, Chaudhuri T, Stefanutti P (2014) Power electronic traction transformer-medium voltage prototype. IEEE Trans Ind Electr 61(7):3257-3268

16. Glinka M, Marquardt R (2005) A new AC/AC multilevel converter family. IEEE Trans Ind Electr 52(3):662-669

17. Winkelnkemper M, Korn A, Steimer P (2010) A modular direct converter for transformerless rail interties. In: 2010 IEEE International Symposium on Industrial Electronics. 4-7 July 2010, Bari

18. Abrahamsson L, Schütte T, Östlund S (2012) Use of converters for feeding of AC railways for all frequencies. Energy Sustain Develop 16(3):368-378

19. Kawahara K, Hisamizu Y, Hase S, Mochinaga Y (2000) Development of static var compensator for San-yo Shinkansen. Q Rep RTRI 41(4):148-153

20. Lee GS, Hwang PI, Lee BG, Moon SI (2020) Loss minimization of electrified railway traction systems using SVC based on particle swarm optimization. IEEE Access 8:219680-219689

21. Kulworawanichpong T, Goodman CJ (2005) Optimal area control of AC railway systems via PWM traction drives. IEE Proc Electr Power Appl 152(1):33-70

22. Raygani SV, Tahavorgar A, Fazel SS, Moaveni B (2012) Load flow analysis and future development study for an AC electric railway. IET Electr Syst Transp 2(3):139-147

23. Hu S, Xie B, Li Y, Gao X, Zhang Z, Luo L, Krause O, Cao Y (2017) A power factor-oriented railway power flow controller for power quality improvement in electrical railway power system. IEEE Trans Ind Electr 64(2):1167-1177

24. Tanta M, Pinto JG, Monteiro V, Martins AP, Carvalho AS, Afonso JL (2020) Topologies and operation modes of rail power conditioners in AC traction grids: Review and comprehensive comparison. Energies 13(9):2151

25. Shu Z, Xie S, Li Q (2011) Single-phase back-to-back converter for active power balancing, reactive power compensation, and harmonic filtering in traction power system. IEEE Trans Power Electr 26(2):334-343

26. Chen M, Li Q, Roberts C, Hillmansen S, Tricoli P, Zhao N, Krastev I (2016) Modelling and performance analysis of advanced combined co-phase traction power supply system in electrified railway. IET Gener Trans Distr 10(4):906-916

27. Huang X, Liao Q, Li Q, Tang S, Sun K (2020) Power management in co-phase traction power supply system with super capacitor energy storage for electrified railways. Railway Eng Sci 28(1):85-96
28. Xie S, Zhang Y, Wang H (2021) A novel co-phase power supply system for electrified railway based on $\mathrm{v}$ type connection traction transformer. Energies 14(4):1214

29. Bueno A, Aller JM, Restrepo JA, Harley R, Habetler TG (2013) Harmonic and unbalance compensation based on direct power control for electric railway systems. IEEE Trans Power Electr 28(12):5823-5831

30. Rodrigues P, Morais VA, Martins A, Carvalho A (2019) STATCOM simulation models for analysis of electrified railways. In: IECON 2019 - 45th Annual Conference of the IEEE Industrial Electronics Society. 14-17 Oct., Lisbon

31. Barros L, Tanta M, Martins A, Afonso J, Pinto J (2021) Evaluation of static synchronous compensator and rail power conditioner in electrified railway systems using v/v and scott power transformers. EAI Endorsed Transactions on Energy Web p. 169164

32. Kondo K (2010) Recent energy saving technologies on railway traction systems. IEEJ Trans Electr Electr Eng 5(3):298-303

33. Park J, Heo J, Shin S, Kim H (2017) Economic evaluation of ESS in urban railway substation for peak load shaving based on net present value. J Electr Eng Technol 12(2):981-987

34. Morais V, Afonso J, Martins A (2021) Towards smart railways: a charging strategy for railway energy storage systems. EAI Endors Trans Energy Web 18(41):168136

35. International Union of Railways (UIC) (2016) Fundación de los Ferrocarriles Españoles (FFE): Technologies and potential developments for energy efficiencyand $\mathrm{CO} 2$ reductions in rail systems

36. Takagi K, Takagi M, Hayashiya H, Iwafune Y (2017) Proposal and effect evaluation of RPC application with energy storage system for regenerative energy utilization of high speed railway. J Int Council Electr Eng 7(1):227-232

37. Gao Z, Lu Q, Wang C, Fu J, He B (2019) Energy-storage-based smart electrical infrastructure and regenerative braking energy management in AC-fed railways with neutral zones. Energies 12(21):4053

38. Hansen I, Pachl J (2014) Railway Timetabling \& Operations. PMC Media House

39. Ho T, Chi Y, Wang J, Leung K, Siu L, Tse C (2005) Probabilistic load flow in AC electrified railways. IEE Proc Electr Power Appl 152(4): 1003

40. Zinser M, Betz T, Becker M, Geilke M, Terschlüsen C, Kaluza A, Johansson I, Warg J (2019) Prism: A macroscopic Monte Carlo railway simulation. In: Proceedings of The 12th World Congress on Railway Research (WCRR). Tokyo

41. Yang S, Wu M (2010) Load probability model of electrified railway traction substation. Automation of Electric Power Systems 34(24):40-45

42. Che Y, Wang X, Lv X, Hu Y, Li L, Chen H (2018) Impact analysis of traction loads on power grid based on probabilistic three-phases load flow. J Eng 2019(16):2306-2311

43. Yang S, Song K, Zhu G (2019) Stochastic process and simulation of traction load for high speed railways. IEEE Access 7:76049-76060

44. Zhang LY, Li QZ, Xing XQ (2009) Probability distribution for the feeder current of traction substation. In: 2009 Asia-Pacific Power and Energy Engineering Conference. 27-31 March, Wuhan

45. Tang K, Li Q, Zhang L (2010) Probability distribution of feeder current of electrified railway traction. pp. 1-4. Chengdu

46. Snider L, Lo E, Lai T (2001) Stochastic power quality study of distribution supply to metro transit railway. pp. 283-288 vol.1. IEEE, Vancouver, BC, Canada

47. Li J, Huang S, Zhao J, Daozhi X (2002) Simulation for probabilistic harmonic currents of electrical railway traction substation. 
In: Proceedings of the International Conference on Power System Technology, vol. 4, pp. 2511-2515. Kunming, China

48. Che Y, Wang X, Lv X, Hu Y, Teng Y (2019) Study on probability distribution of electrified railway traction loads based on kernel density estimator via diffusion. Int J Electr Power Energy Syst 106:383-391

49. de la Fuente EP, Jiménez-Octavio J, Rodriguez-Pecharroman R, Lopez AL (2011) Stochastic traffic generator for monte carlo load flow simulation. WIT Transactions on Modelling and Simulation 51
50. Brandimarte P (2014) Handbook in Monte Carlo Simulation: Applications in Financial Engineering, Risk Management, and Economics. Wiley, pp 1-10

51. Öner M, Kocakoç ID (2017) JMASM 49: a compilation of some popular goodness of fit tests for normal distribution: their algorithms and MATLAB codes (MATLAB). J Mod Appl Stat Methods 16(2):547-575 